OPEN ACCESS

Edited by:

Aditi Banerjee,

University of Maryland, Baltimore,

United States

Reviewed by:

Timothy Price,

The Queen Elizabeth Hospital (TQEH),

Australia

Barbara Geerinckx

TQEH, Adelaide, Australia, in

collaboration with reviewer TP

Chitta Ranjan Sahu,

University of Kalyani, India

*Correspondence:

Jeongseon Kim

jskim@ncc.re.kr

Specialty section:

This article was submitted to

Gastrointestinal Cancers:

Colorectal Cancer,

a section of the journal

Frontiers in Oncology

Received: 19 August 2021 Accepted: 31 December 2021 Published: 09 February 2022

Citation:

Hoang T, Sohn DK, Kim BC, Cha Y and Kim J (2022) Efficacy and Safety of Systemic Treatments Among Colorectal Cancer Patients:

A Network Meta-Analysis of

Randomized Controlled Trials.

Front. Oncol. 11:756214.

doi: 10.3389/fonc.2021.756214

\section{Efficacy and Safety of Systemic Treatments Among Colorectal Cancer Patients: A Network Meta-Analysis of Randomized Controlled Trials}

\author{
Tung Hoang ${ }^{1}$, Dae Kyung Sohn ${ }^{2}$, Byung Chang Kim ${ }^{2}$, Yongjun $\mathrm{Cha}^{2}$ and Jeongseon Kim ${ }^{1 *}$ \\ ${ }_{1}^{1}$ Department of Cancer Biomedical Science, National Cancer Center Graduate School of Cancer Science and Policy, \\ Goyang, South Korea, ${ }^{2}$ Center for Colorectal Cancer, Research Institute and Hospital, National Cancer Center, Goyang, \\ South Korea
}

Background: Systemic treatments, namely, either monotherapy or combination therapy, are commonly administered to patients with advanced or metastatic colorectal cancer $(\mathrm{CRC})$. This study aimed to provide the complete efficacy and safety profiles and ranking of systemic therapies for the treatment of unresectable advanced or metastatic CRC.

Methods: We searched PubMed, Embase, the Cochrane Library, and ClinicalTrials.gov from inception until June 30, 2021, and also the bibliographies of relevant studies. Randomized controlled trials comparing two or more treatments, namely, at least capecitabine, 5-fluorouracil, leucovorin, irinotecan, bevacizumab, cetuximab, oxaliplatin, or panitumumab were investigated. A network meta-analysis using the Bayesian approach was performed to compare the efficacy and safety of treatments. The surface under the cumulative ranking curve (SUCRA) was calculated for the probability of each treatment as the most effective. The overall response rate (ORR), disease control rate (DCR), overall survival (OS), progression-free survival (PFS), adverse events (AEs) grade $\geq 3$, and serious adverse events (SAEs) were evaluated.

Results: One hundred two publications with 36,147 participants were assigned to 39 different treatments. Among 11 treatments with full information on six outcomes, FOLFIRI/ FOLFOX/FOLFOXIRI + bevacizumab significantly improved both the ORR and DCR, compared to FOLFIRI. Although FOLFOX and FOLFIRI/FOLFOX + cetuximab significantly prolonged both OS and PFS, treatments were comparable in terms of AEs grade $\geq 3$ and SAEs. The top highest SUCRA values were observed in the FOLFOXIRI + panitumumab group for ORR (96\%) and DCR (99\%), FOLFIRI + bevacizumab + panitumumab group for OS (62\%) and PFS (54\%), and FOLFOXIRI + bevacizumab group for AEs grade $\geq 3$ (59\%) and SAEs (59\%) outcomes. 
Conclusions: These findings suggest an available range of systemic treatment therapies with different efficacy and safety profiles with patients. Further investigations of the side effects and mutation status are required to confirm our findings.

Systematic Review Registration: https://www.crd.york.ac.uk/prospero/, identifier CRD42019127772

Keywords: network meta-analysis, colorectal cancer, metastasis, chemotherapy, targeted therapy

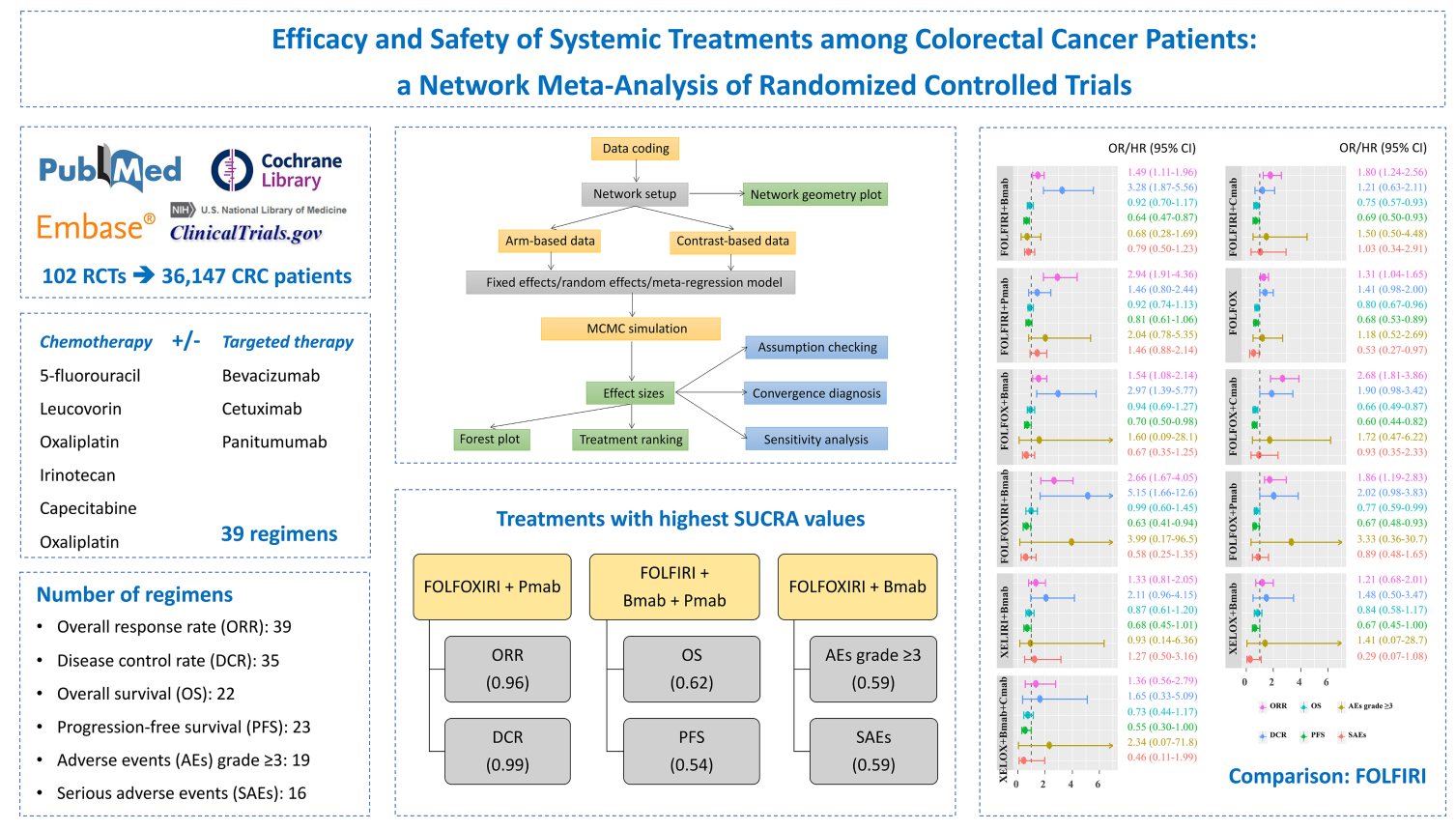

\section{INTRODUCTION}

According to the most recent update in 2018, colorectal cancer (CRC) is still the third most common type of cancer and the second leading cause of cancer-related deaths worldwide (1). Currently, tumor resection is recommended for individuals with stage I, II, and III CRC (2). Additionally, adjuvant therapy is normally administered after completing surgery in an attempt to eliminate residual micrometastatic disease, which leads to decreased tumor recurrence and improved prolonged survival rate (3). When surgery is rarely indicated for unresectable metastatic CRC, several chemotherapy regimens are accepted for treatment (4). Among patients who are appropriate for intensive

Abbreviations: CRC, colorectal cancer; NMA, network meta-analysis; OR, odds ratio; HR, hazard ratio; CrI, credible interval; ORR, overall response rate; DCR, disease control rate; OS, overall survival; PFS, progression-free survival; AEs, adverse events; SAEs, serious adverse events; FOLFIRI, 5-fluorouracil/leucovorin/ irinotecan; FOLFOX, 5-fluorouracil/leucovorin/oxaliplatin; FOLFOFIRI, 5fluorouracil/leucovorin/oxaliplatin/irinotecan; XELOX, capecitabine/oxaliplatin; XELIRI, capecitabine/irinotecan; 5-FU, 5-fluorouracil; Bmab, bevacizumab; Cmab, cetuximab; Pmab, panitumumab. therapy with RAS mutations, 5-fluorouracil (5-FU) + leucovorin, 5-FU/leucovorin/oxaliplatin (FOLFOX), 5-FU/leucovorin/ oxaliplatin/irinotecan (FOLFOXIRI), and capecitabine/ oxaliplatin (XELOX) can be used alone or in combination with bevacizumab (Bmab) (4). For patients with a wild-type RAS genotype, cetuximab or panitumumab is combined with FOLFOX or 5-FU/leucovorin/irinotecan (FOLFIRI) (4). Infusions of $5-\mathrm{FU}+$ leucovorin \pm bevacizumab, and cetuximab $(\mathrm{Cmab})$ or panitumumab (Pmab) are indicated for individuals for whom intensive therapy is not recommended and present with mutated and RAS wild-type genotypes, respectively (4).

A network meta-analysis (NMA) has been used to simultaneously compare multiple treatments by combining direct evidence from head-to-head or controlled trials and indirect results within a net-like relation to provide network evidence (5). Recently, an NMA focused on the efficacy of 17 regimens that do not differentiate the specific chemotherapies, especially capecitabine, 5-FU, leucovorin, irinotecan, and oxaliplatin, in each treatment comparison (6). Another study evaluated the efficacy of 10 regimens for first-line chemotherapy in patients with advanced CRC; however, their safety profiles have not been investigated (7). Therefore, by performing an 
NMA of randomized controlled trials (RCTs), we aimed to investigate the comparative efficacy and safety of several treatment regimens in patients with advanced or metastatic CRC. The results of the study are expected to provide reliable guidance for the selection of drugs in the treatment of CRC.

\section{MATERIALS AND METHODS}

\section{Search Strategy}

This systematic search was conducted according to the Preferred Reporting Items for Systematic Reviews and Meta-Analyses (PRISMA) guidelines (8). We searched the PubMed, Embase, the Cochrane Library, and ClinicalTrials.gov databases from inception until June 30, 2021. The search was limited to human subjects and clinical trials. We also reviewed the bibliographies of relevant articles to identify additional studies related to the topic.

\section{Study Selection}

The keywords used for the literature search were as follows: treatment ('capecitabine', '5-fluorouracil', 'leucovorin', 'irinotecan', 'bevacizumab', 'cetuximab', 'oxaliplatin', and 'panitumumab') and CRC ('colon cancer', 'rectal cancer', and 'colorectal cancer'). We included RCTs that (1) recruited patients with advanced or metastatic CRC; (2) investigated the efficacy and/or safety of combination therapies containing at least one regimen from the search; and (3) measured outcomes such as the hazard ratio (HR) for overall survival (OS) and progression-free survival (PFS), the overall response rate (ORR), the disease control rate (DCR), adverse events (AEs) grade $\geq 3$, and serious adverse events (SAEs). Duplicate publications from the same study population were excluded.

Two authors independently reviewed the studies, discussed any controversies related to study selection, and extracted the information from the selected studies.

\section{Identification}

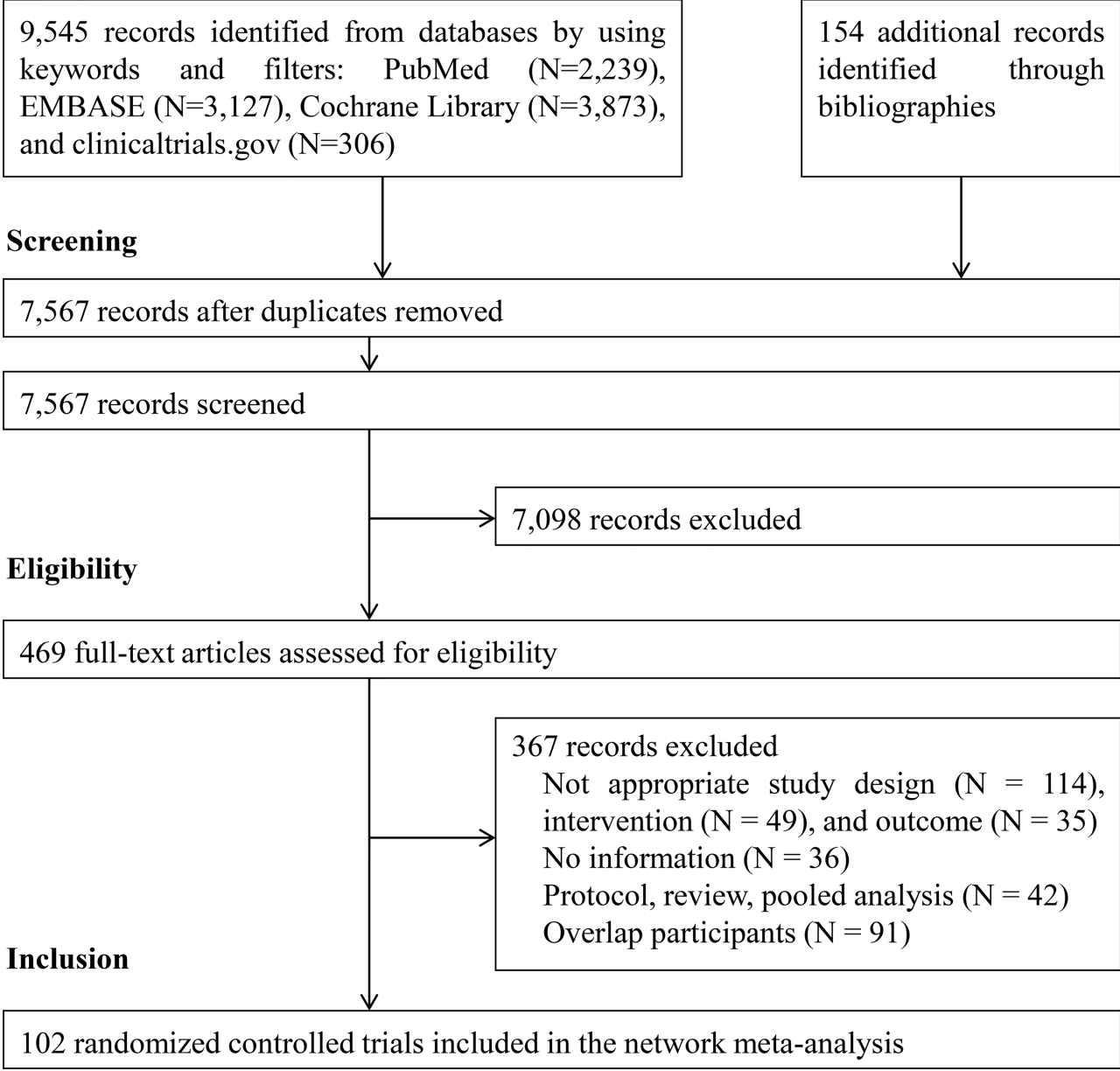

FIGURE 1 | PRISMA flowchart for study selection. 


\section{Quality Assessment}

The risk of bias for eligible studies was independently evaluated by two investigators in accordance with the Cochrane Handbook for Systematic Reviews of Intervention (9). Any discrepancies were discussed and resolved by consulting with other coauthors.

\section{Statistical Analysis}

The pooled HRs for OS and PFS, the odds ratios (ORs) for the ORR, the DCR, AEs grade $\geq 3$, and SAEs and their $95 \%$ credible intervals (CrIs) were calculated to evaluate the differences among regimens.

The node-splitting statistic was applied to assess the inconsistency assumption between direct pairwise metaanalyses and indirect estimates (10). The $I^{2}$ value was calcualated to test the heterogeneity among studies (10).

We applied the generalized linear model for Bayesian NMA, and the results of the random effects model were compared with those of the fixed effects model to compute the pooled estimates of outcomes (11). The treatment line was additionally considered as a covariate in the meta-regression model of the sensitivity analysis (12). Additionally, the convergence diagnosis of the Markov chain Monte Carlo (MCMC) with 50,000 burn-in iterations and 3 chains was used to obtain robust results. Detailed descriptions of the method are provided in the Supplementary Appendix. MCMC simulation analyses were performed using WinBUGS 1.4.3 software (MRC Biostatistics Unit, UK) with the Bayesian framework.

We determined the probabilities of being primary and secondary therapies for each treatment and the surface under the cumulative ranking curve (SUCRA), which ranges between 0 and $100 \%$, for each outcome to calculate the probability of each treatment being the most effective (13). A higher SUCRA value indicates a greater likelihood that the treatment is closer to the top rank; in contrast, a lower SUCRA value indicates a greater likelihood that the treatment is closer to the bottom rank (14). The Spearman method was applied to calculate the pairwise correlation of outcomes, using the 'psych' R package (15). Enhanced k-means cluster analyses were used to group similar treatments with the 'factoextra' R package (16).

Detailed descriptions of the methodology are presented in the eMethods. The study methodology and progress were registered and approved by the National Institute for Health ResearchInternational Prospective Register of Systematic Reviews (PROSPERO registration number: CRD42019127772).

\section{RESULTS}

\section{Selection of Eligible Studies}

Figure 1 illustrates the PRISMA flowchart for the literature search and screening results. By searching four electronic databases and reviewing the bibliographies of five relevant cost-effectiveness analyses and NMAs, we identified 9,699 candidate reports. After screening the abstracts and titles, 469 articles remained and the full texts were assessed. Ultimately, 102 citations (92 main RCTs and 10 subgroup publications for additional outcome results) were included in the NMA (17-118).

\section{Characteristics of the Included Studies}

Of all 94 main phase II/III RCTs, three were three-arm studies. The number of males was higher than the number of females in each research population, and the median age ranged between 60 and 70 years (Supplementary Table S1). Thirty-nine different regimens containing at least capecitabine, 5-FU, leucovorin, irinotecan, oxaliplatin, Bmab, Cmab, and Pmab were identified, and 39 treatments with 36,147 patients were included in the final analysis after excluding treatments not connected to the network (Supplementary Table S2).

\section{Cochrane Risk of Bias Assessment}

In the Cochrane risk of bias assessment, most of the RCTs originated from multicenter trials in which randomization was stratified by different baseline factors, thus minimizing selection bias. In contrast, an open-label design led to a high or unclear risk of performance bias in all the RCTs (Figures 2, 3).

\section{Network Geometry of Available Evidence}

Supplementary Figure S1 shows the network geometry of all the available comparisons of treatments to explore their relationships and contributions. Among them, treatments for which data on specific outcomes were not available or did not contribute to the main network were excluded (Supplementary Table S3). As a result, pairwise comparisons of $39,35,22,23,19$, and 16 systemic therapies regarding the ORR, the DCR, OS, PFS, AEs grade $\geq 3$, and SAEs, respectively, were eligible for the final analysis (Figure 4).

\section{Forest Plots of the Pooled Estimates of 11 Regimens}

The comparative treatment effects of 11 regimens for which the results were available on six outcomes relative to the FOLFIRI combination are shown in Figure 5. Regarding the ORR, FOLFIRI + Bmab/Cmab/Pmab, FOLFOX, FOLFOX + Bmab/ $\mathrm{Cmab} / \mathrm{Pmab}$, and FOLFOXIXI + Bmab resulted in a significant improvement, with ORs (95\% CrIs) of 1.49 (1.11-1.96), 1.80 (1.24-2.56), 2.94 (1.91-4.36), 1.31 (1.04-1.65), 1.54 (1.08-2.14), 2.68 (1.81-3.86), $1.86(1.19-2.83)$, and $2.66(1.67-4.05)$, respectively. Regarding the DCR, the ORs (95\% CrIs) of FOLFIRI/FOLFOX/FOLFOXIRI + Bmab compared to FOLFIRI were 3.28 (1.87-5.56), 2.97 (1.39-5.77), and 5.15 (1.66-12.6), respectively. Regarding survival outcomes, FOLFIRI + Cmab $(\mathrm{HR}=0.75,95 \% \mathrm{CrI}=0.57-0.93$ for OS and $\mathrm{HR}=0.69,95 \% \mathrm{CrI}=0.50-0.93$ for PFS $)$, FOLFOX $(\mathrm{HR}=$ $0.80,95 \% \mathrm{CrI}=0.67-0.96$ for $\mathrm{OS}$ and $\mathrm{HR}=0.68,95 \% \mathrm{CrI}=0.53-$ 0.89 for PFS), FOLFOX + Cmab ( HR $=0.66,95 \% \mathrm{CrI}=0.49-0.87$ for $\mathrm{OS}$ and $\mathrm{HR}=0.60,95 \% \mathrm{CrI}=0.44-0.82$ for $\mathrm{PFS})$, and FOLFOX + Pmab (HR $=0.77,95 \%$ CrI $=0.59-0.99$ for OS and $\mathrm{HR}=0.67,95 \% \mathrm{CrI}=0.48-0.93$ for PFS) prolonged both OS and PFS compared with FOLFIRI. PFS was significantly longer in patients treated with the FOLFIRI + Bmab, FOLFOX + Bmab, and FOLFOXIRI + Bmab regimens (36, 30, and 37\%, respectively) than in patients treated with the FOLFIRI regimen. In terms of safety endpoints, including AEs grade $\geq 3$ and SAEs, the treatment effects were comparable between the 11 treatments and FOLFIRI; however, a $47 \%$ lower probability of SAEs was observed in the FOLFOX treatment group relative to 


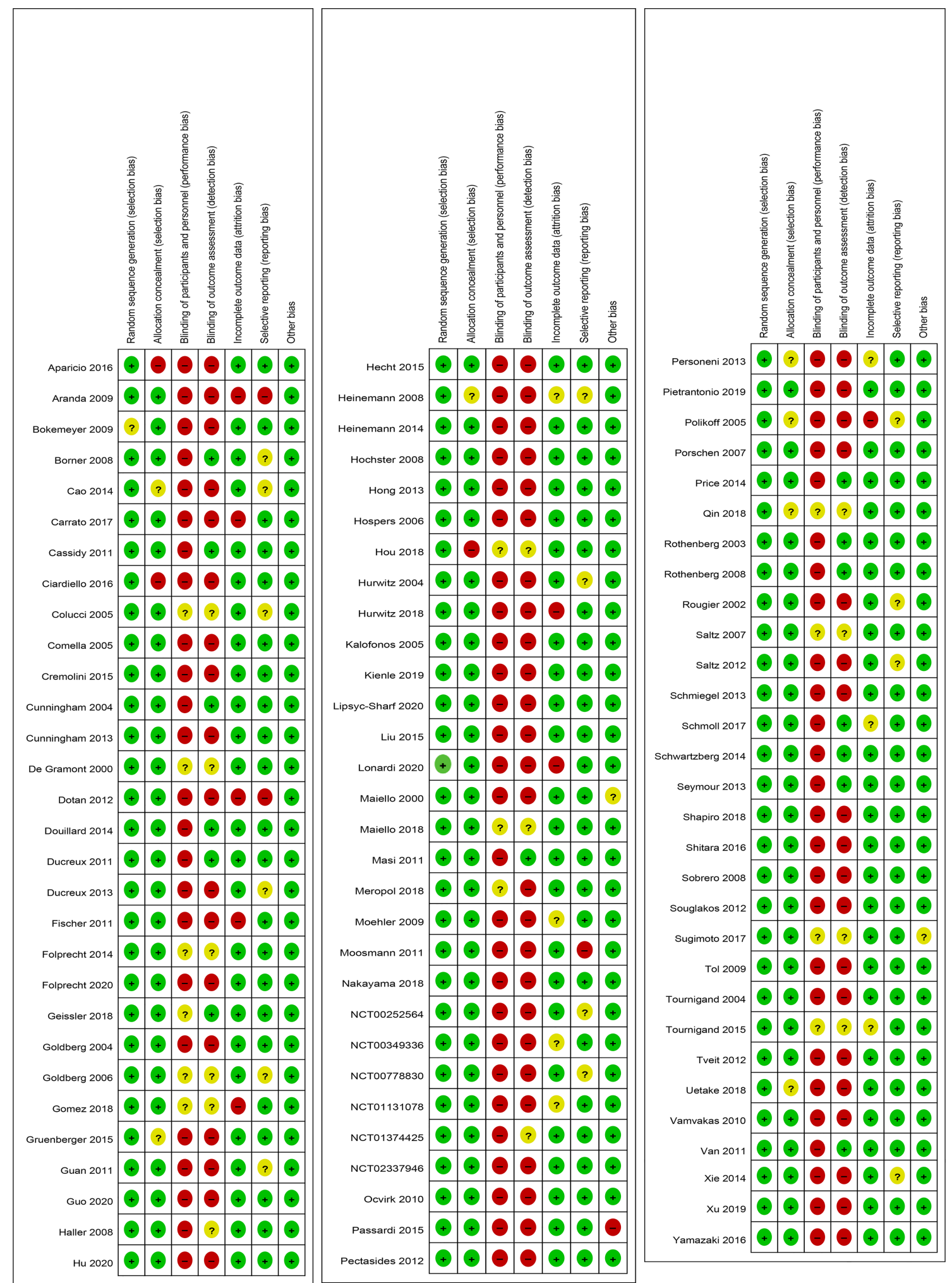

FIGURE 2 | Risk of bias summary for each included study. 


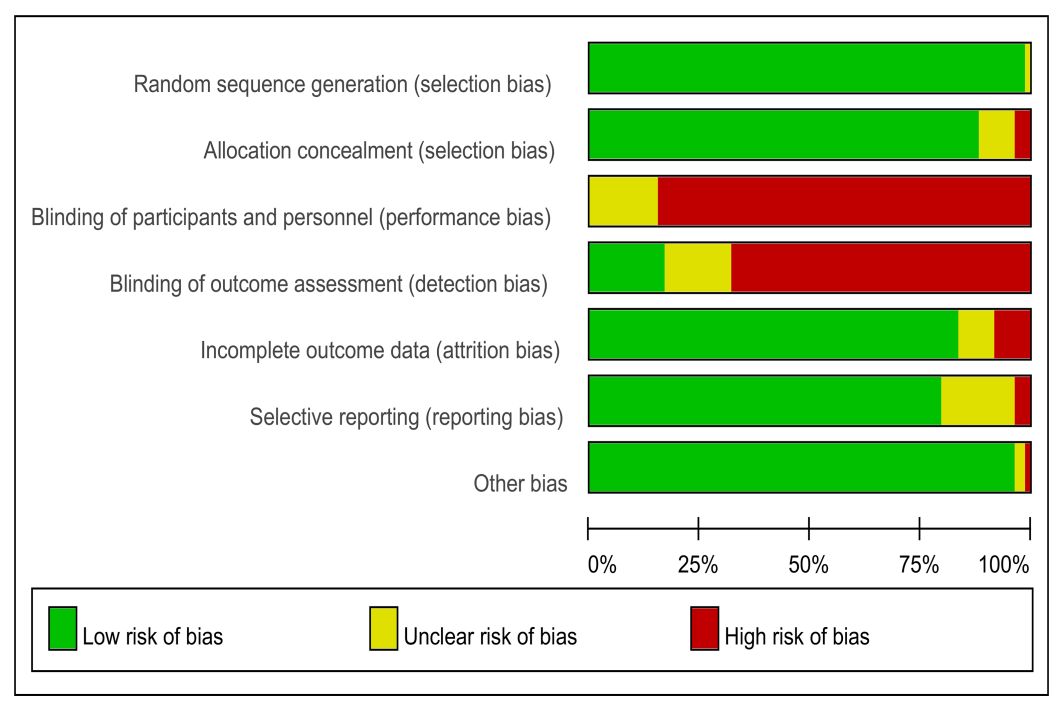

FIGURE 3 | Risk of bias graph across all the included studies.

the FOLFIRI treatment group $(\mathrm{OR}=0.53,95 \% \mathrm{CrI}=0.27-0.97)$. The complete results for pairwise comparisons of all the six outcomes are available in Supplementary Tables S4-S6.

\section{Inconsistency and Heterogeneity Assumption}

In the inconsistency analysis, no significant differences were observed between direct and indirect estimates of PFS, AEs grade $\geq 3$, and SAEs outcomes (Supplementary Table S7). The statistical inconsistency between direct and indirect estimates of the ORR for FOLFIRI + Pmab vs FOLFIRI + Bmab $(\mathrm{p}=0.01)$ and FOLFOX + Pmab vs FOLFIRI + Pmab ( $\mathrm{p}=0.01)$; the DCR for FOLFOX + Pmab vs. FOLFOX ( $\mathrm{p}=0.04)$, XELOX + Cmab vs. XELOX ( $p=0.01)$, XELOX + Bmab + Cmab vs. XELOX + Bmab ( $\mathrm{p}=0.01)$, and XELOX + Bmab + Cmab vs. XELOX + Cmab ( $p=0.004)$, and the OS for FOLFOXIRI + Bmab vs. FOLFIRI + Bmab $(p=0.01)$ and FOLFOXIRI + Bmab vs. FOLFOX + Bmab $(\mathrm{p}=0.01)$ was observed in the nodesplitting model. The between-trial variance is reported as the global $I^{2}$ value in both pairwise and consistency estimates, and the substantial heterogeneity is reported as a measure of ORR $\left(I^{2}=39 \%\right)$, DCR $\left(I^{2}=32 \%\right)$, OS $\left(I^{2}=30 \%\right)$, PFS $\left(I^{2}=69 \%\right)$, and AEs grade $\geq 3\left(I^{2}=84 \%\right)$ for pairwise $I^{2}$ and ORR $\left(I^{2}=38 \%\right)$, DCR $\left(I^{2}=49 \%\right)$, OS $\left(I^{2}=53 \%\right)$, PFS $\left(I^{2}=70 \%\right)$, and AEs grade $\geq 3\left(I^{2}=81 \%\right)$ for consistent $I^{2}$ (Supplementary Table S8).

\section{Sensitivity Analysis}

The pooled ORs/HRs for all the effect sizes from the random effects model were compared with the those of the fixed effects model and the meta-regression of treatment line covariates in a scatter plot with the slope of the straight line equal to one. The results were similar among the three models, except for some estimates of ORR and DCR outcomes for the comparison of the random-effects model with both fixed-effects and meta- regression models (Figure 6). Thus, the estimates obtained after including the treatment line in the meta-regression model did not differ substantially from those in the randomeffects model.

Parameters for the comparison of model performance of fixed-effects, random-effects, and meta-regression models are shown in Table 1. Overall, the deviance information criteria values in the random effects models were relatively lower than those in the fixed effects and meta-regression models, which suggested better model performance.

\section{Treatment Ranking Probability and SUCRA Clustering Analysis}

Figure 7 and Supplementary Table $\mathbf{S 9}$ show the probabilities of each regimen being used as primary and secondary options for the treatment of advanced or metastatic CRC. Compared to the other regimens, FOLFOXIRI + Pmab had the highest probability of becoming a first-line candidate that improved both the ORR (43\%) and the DCR (86\%). In addition, XELOX + Cmab and FOLFOXIRI + Bmab were most likely to be second-line therapies in terms of improving the ORR (26\%) and DCR (47\%), respectively. When considering OS and PFS, capecitabine + Bmab had the highest probability of becoming a first-line candidate, with ranking probabilities of $56 \%$ for OS and $39 \%$ for PFS. Furthermore, capecitabine and FOLFIRI + Bmab + Pmab were considered to be the second-line candidates for prolonging OS (36\%) and PFS (19\%), respectively, and had the highest probability of becoming a first-line candidate for prolonging OS (56\%) and PFS (39\%). A similar trend was observed for AEs grade $\geq 3$, with a value of $29 \%$ for oxaliplatin followed by $21 \%$ for leucovorin +5 -FU as a first-line therapy and $26 \%$ for leucovorin +5 -FU and $16 \%$ for oxaliplatin as a secondline therapy. Although XELOX + Bmab had the greatest probability of decreasing the number of SAEs when used as a 


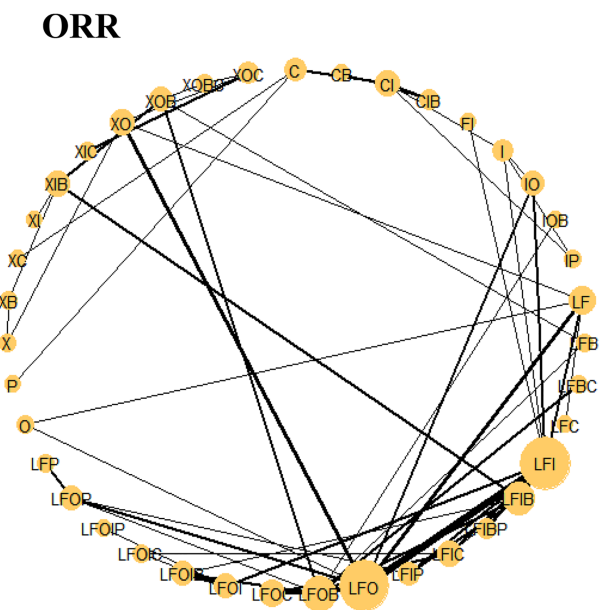

OS

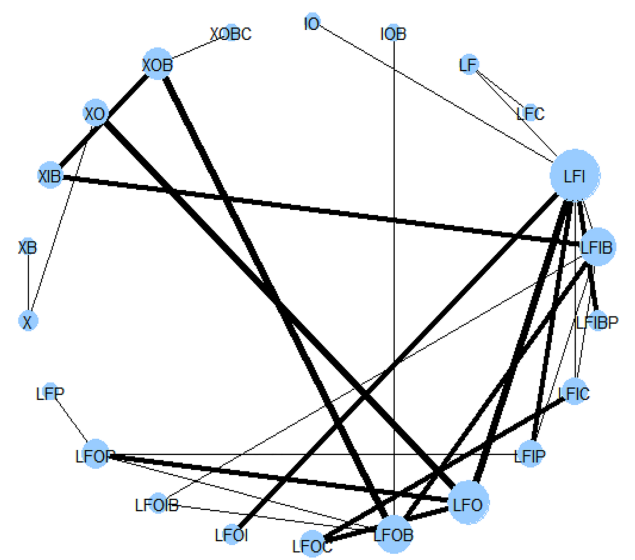

AEs grade $\geq 3$

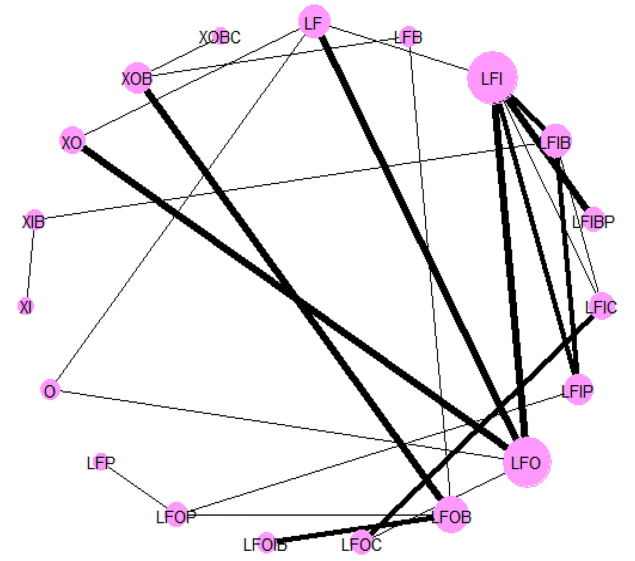

DCR

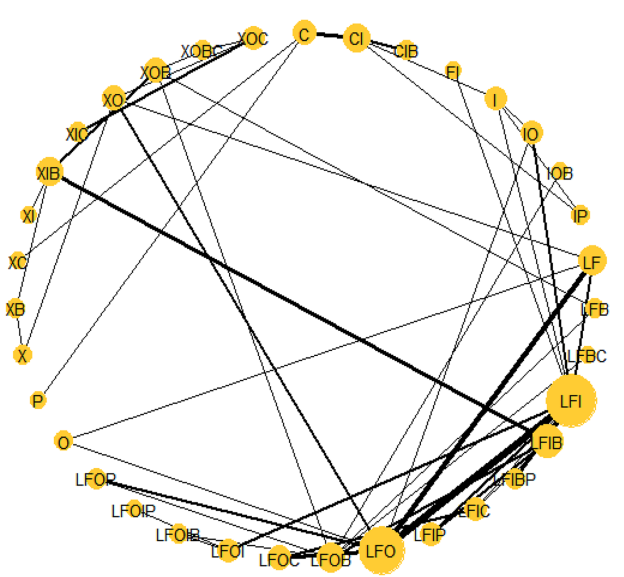

PFS

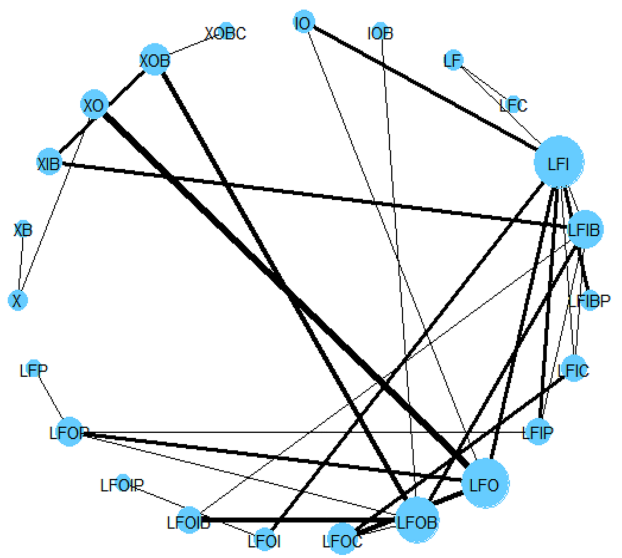

SAES

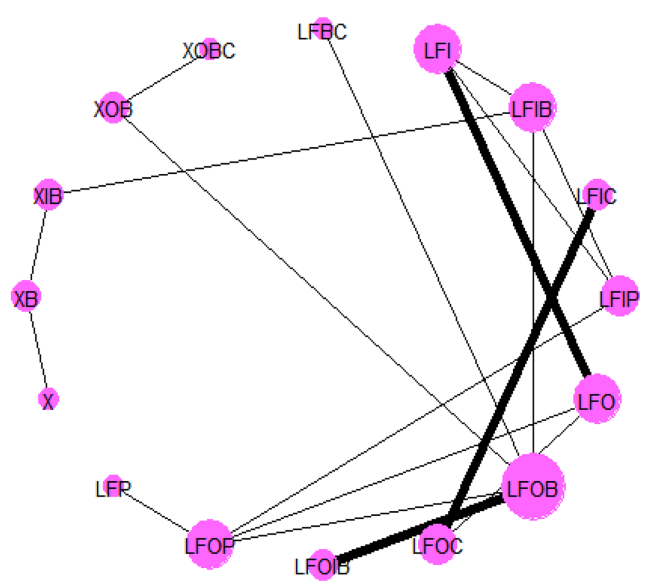

FIGURE 4 | Network geometry of treatments included in the final analysis. ORR, overall response rate; DCR, disease control rate; OS, overall survival; PFS, progression-free survival; AEs, adverse events; SAEs, serious adverse events; X, capecitabine; B, bevacizumab; C, cetuximab; F, 5-fluorouracil; I, irinotecan; L, leucovorin; O, oxaliplatin; P, panitumumab. The network plots are based on treatments in a connected network according to different types of outcomes. The size of the node indicates the number of participants receiving each treatment, and the thickness of the line illustrates the number of studies included each comparison. 


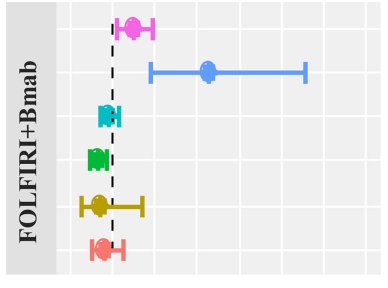

$1.49(1.11-1.96)$

$3.28(1.87-5.56)$

$0.92(0.70-1.17)$

$0.64(0.47-0.87)$

$0.68(0.28-1.69)$

$0.79(0.50-1.23)$

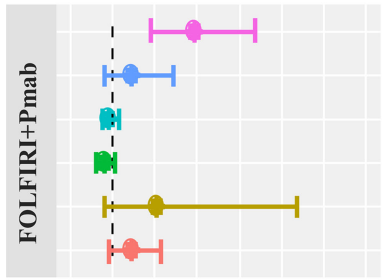

$2.94(1.91-4.36)$

$1.46(0.80-2.44)$

$0.92(0.74-1.13)$

$0.81(0.61-1.06)$

$2.04(0.78-5.35)$

$1.46(0.88-2.14)$

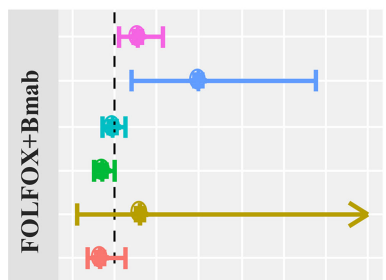

$1.54(1.08-2.14)$

$2.97(1.39-5.77)$

$0.94(0.69-1.27)$

$0.70(0.50-0.98)$

$1.60(0.09-28.1)$

$0.67(0.35-1.25)$

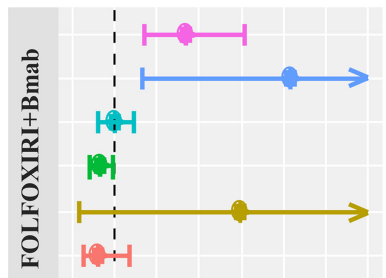

$2.66(1.67-4.05)$

$5.15(1.66-12.6)$

$0.99(0.60-1.45)$

$0.63(0.41-0.94)$

$3.99(0.17-96.5)$

$0.58(0.25-1.35)$

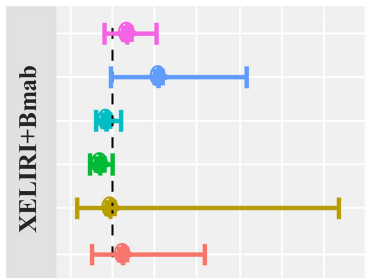

$1.33(0.81-2.05)$

$2.11(0.96-4.15)$

$0.87(0.61-1.20)$

$0.68(0.45-1.01)$

$0.93(0.14-6.36)$

$1.27(0.50-3.16)$

$1.36(0.56-2.79)$

$1.65(0.33-5.09)$

$0.73(0.44-1.17)$

$0.55(0.30-1.00)$

$2.34(0.07-71.8)$

$0.46(0.11-1.99)$

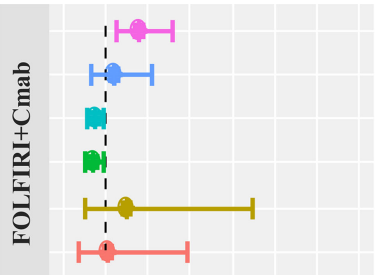

$1.80(1.24-2.56)$

$1.21(0.63-2.11)$

$0.75(0.57-0.93)$

$0.69(0.50-0.93)$

$1.50(0.50-4.48)$

$1.03(0.34-2.91)$

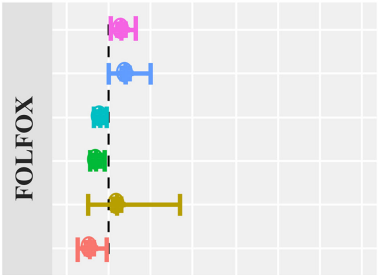

$1.31(1.04-1.65)$

$1.41(0.98-2.00)$

$0.80(0.67-0.96)$

$0.68(0.53-0.89)$

$1.18(0.52-2.69)$

$0.53(0.27-0.97)$

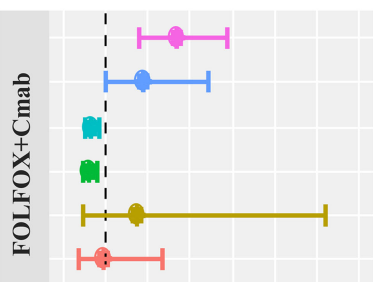

$2.68(1.81-3.86)$

$1.90(0.98-3.42)$

$0.66(0.49-0.87)$

$0.60(0.44-0.82)$

$1.72(0.47-6.22)$

$0.93(0.35-2.33)$

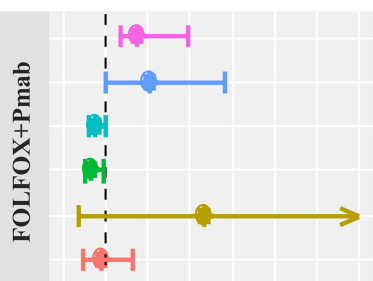

$1.86(1.19-2.83)$

$2.02(0.98-3.83)$

$0.77(0.59-0.99)$

0.67 (0.48-0.93)

$3.33(0.36-30.7)$

$0.89(0.48-1.65)$

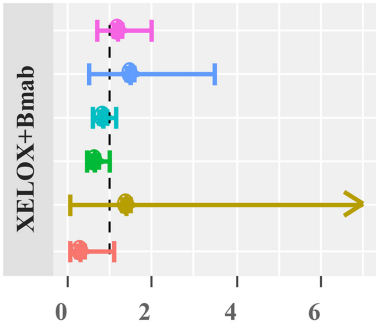

$1.21(0.68-2.01)$

$1.48(0.50-3.47)$

$0.84(0.58-1.17)$

$0.67(0.45-1.00)$

$1.41(0.07-28.7)$

$0.29(0.07-1.08)$

+ ORR $\rightarrow$ OS AEs grade $\geq 3$

+ DCR + PFS + SAEs

FIGURE 5 | Scatter plot showing differences in estimates obtained from fixed-effects, random-effects, and meta-regression models. ORR, overall response rate; $\mathrm{DCR}$, disease control rate; OS, overall survival; PFS, progression-free survival; AEs, adverse events; SAEs, serious adverse events; FOLFIRI, 5-fluorouracil/leucovorin/ irinotecan; FOLFOX, 5-fluorouracil/leucovorin/oxaliplatin; FOLFOFIRI, 5-fluorouracil/leucovorin/oxaliplatin/irinotecan; XELOX, capecitabine/oxaliplatin; XELIRI, capecitabine/irinotecan; Bmab, bevacizumab; Cmab, cetuximab; Pmab, panitumumab.

primary treatment $(62 \%)$, XELOX + Bmab + Cmab was suggested as a secondary therapy (36\%).

The SUCRA value, which summarizes the cumulative ranking of the treatment line, was calculated for each treatment (Table 2).
The highest SUCRA values were observed for ORR (96\%) and DCR (99\%) in patients treated with FOLFOXI + Pmab, OS (62\%) and PFS (54\%) in patients treated with FOLFIRI + Bmab + Pmab, and AEs grade $\geq 3(59 \%)$ and SAEs (59\%) in patients 


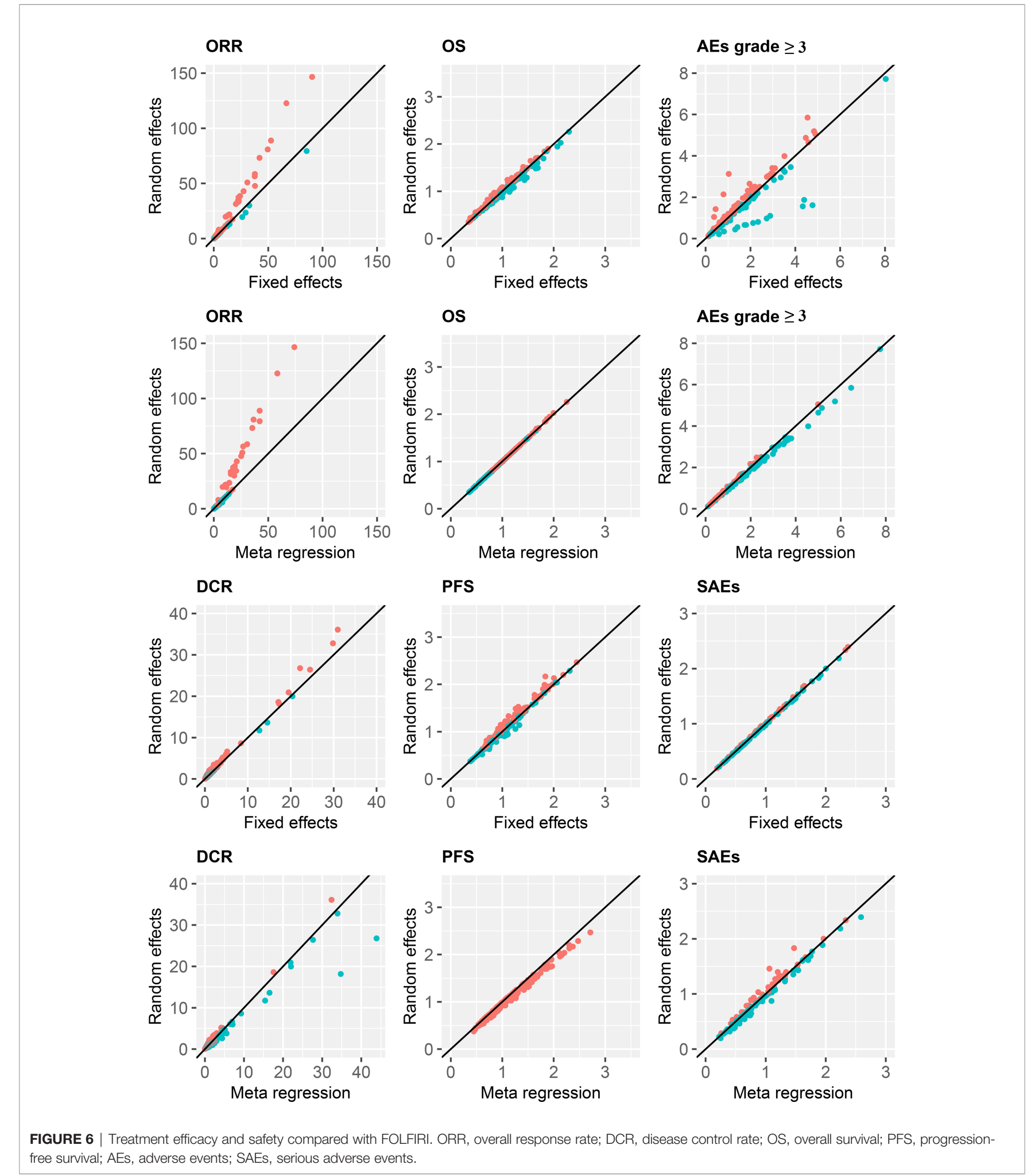

treated with FOLFOXIRI + Bmab. Figure 8 displays the association between the SUCRA value and each pair of outcomes. Substantial Spearman's correlations were observed between the SUCRA values for ORR and DCR (0.61), ORR and OS (0.67), and AEs grade $\geq 3$ and SAEs (0.61). The enhanced $\mathrm{k}$-means cluster analysis showed that the groups of treatments exerted a similar pairwise effect on the outcomes. Overall, treatments with higher SUCRA values were located in the 
TABLE 1 | Parameters used for model selection.

\begin{tabular}{|c|c|c|c|c|}
\hline Outcome & Parameter & Fixed effects & Random effects & Meta-regression \\
\hline \multirow[t]{5}{*}{ Overall response rate } & Posterior mean residual deviance, $\bar{D}_{\text {res }}$ & 253 & 203 & 202.1 \\
\hline & Posterior mean deviances, $\bar{D}_{\text {model }}$ & $1,179.01$ & $1,128.59$ & $1,128.44$ \\
\hline & Effective number of parameters, $p_{D}$ & 133.36 & 160.774 & 161.692 \\
\hline & Between-study standard deviation, $\sigma$ & & 0.283 & 0.286 \\
\hline & Deviance information criteria, $D / C$ & $1,312.37$ & $1,289.36$ & $1,290.13$ \\
\hline \multirow[t]{5}{*}{ Disease control rate } & Posterior mean residual deviance, $\bar{D}_{\text {res }}$ & 203.4 & & 160.2 \\
\hline & Posterior mean deviances, $\bar{D}_{\text {model }}$ & 860.277 & 811.14 & 816.581 \\
\hline & Effective number of parameters, $p_{D}$ & 104.06 & 126.235 & 123.259 \\
\hline & Between-study standard deviation, $\sigma$ & & 0.409 & 0.316 \\
\hline & Deviance information criteria, $D I C$ & 964.337 & 937.374 & 939.84 \\
\hline \multirow[t]{5}{*}{ Overall survival } & Posterior mean residual deviance, $\bar{D}_{\text {res }}$ & 69.15 & 50.93 & 51.3 \\
\hline & Posterior mean deviances, $\bar{D}_{\text {model }}$ & -21.076 & -39.326 & -38.918 \\
\hline & Effective number of parameters, $p_{D}$ & 20.992 & 32.378 & 32.296 \\
\hline & Between-study standard deviation, $\sigma$ & & 0.017 & 0.026 \\
\hline & Deviance information criteria, DIC & -0.084 & -6.948 & -6.622 \\
\hline \multirow[t]{5}{*}{ Progression-free survival } & Posterior mean residual deviance, $\bar{D}_{\text {res }}$ & 109.2 & 51.25 & 51.22 \\
\hline & Posterior mean deviances, $\bar{D}_{\text {model }}$ & 1.442 & -56.466 & -56.488 \\
\hline & Effective number of parameters, $p_{D}$ & 21.982 & 40.924 & 40.944 \\
\hline & Between-study standard deviation, $\sigma$ & & 0.193 & 0.193 \\
\hline & Deviance information criteria, $D / C$ & 23.424 & -15.542 & -15.544 \\
\hline \multirow[t]{5}{*}{ Adverse events grade $\geq 3$} & Posterior mean residual deviance, $\bar{D}_{\text {res }}$ & 186.3 & 71.62 & 71.53 \\
\hline & Posterior mean deviances, $\bar{D}_{\text {model }}$ & 537.81 & 423.174 & 423.094 \\
\hline & Effective number of parameters, $p_{D}$ & 52.067 & 68.344 & 68.582 \\
\hline & Between-study standard deviation, $\sigma$ & & 0.742 & 0.768 \\
\hline & Deviance information criteria, DIC & 589.877 & 491.519 & 491.676 \\
\hline \multirow[t]{5}{*}{ Serious adverse events } & Posterior mean residual deviance, $\bar{D}_{\text {res }}$ & 39.09 & 39.65 & 39.44 \\
\hline & Posterior mean deviances, $\bar{D}_{\text {model }}$ & 250.384 & 250.95 & 250.741 \\
\hline & Effective number of parameters, $p_{D}$ & 36.036 & 37.597 & 38.364 \\
\hline & Between-study standard deviation, $\sigma$ & & 0.136 & 0.159 \\
\hline & Deviance information criteria, DIC & 286.42 & 288.547 & 289.106 \\
\hline
\end{tabular}

upper right quadrant of the plot; in contrast, those with lower SUCRA values were located in the lower left quadrant of the plot.

\section{DISCUSSION}

This study investigated the comparative efficacy and safety of different regimens used to treat advanced or metastatic CRC. The pairwise treatment effects of 39, 35, 22, 23, 19, and 16 systemic therapies on the ORR, DCR, OS, PFS, AEs grade $\geq 3$, and SAEs were compared using a comprehensive NMA. Compared with the FOLFIRI regimen, which was administered to the greatest number of participants, the other 9 treatments, namely, FOLFIRI + (Bmab or Cmab or Pmab), FOLFOX \pm $\mathrm{Bmab} / \mathrm{Cmab} / \mathrm{Pmab}$, and XELIRI/XELOX + Bmab, significantly improved at least one outcome. The highest probabilities of being used as a first-line treatment to improve ORR, DCR, OS, PFS, AEs grade $\geq 3$, and SAEs were reported for FOLFOXIRI + Pmab, FOLFOXIRI + Pmab, capecitabine + Bmab, capecitabine + Bmab, oxaliplatin, and XELOX + Bmab, respectively.

In this study, the replacement of irinotecan in the FOLFIRI regimen by oxaliplatin in the FOLFOX combination resulted in a higher efficacy of prolonging OS and PFS but a lower safety profile of SAEs. Although the efficacy and safety of XELIRI vs. XELOX, XELIRI + Bmab vs. XELOX + Cmab, and irinotecan vs. oxaliplatin were not evaluated, the treatment effects of other irinotecan-based vs. oxaliplatin-based regimens, such as FOLFIRI + Bmab/Cmab/Pmab vs. FOLFOX + Bmab/Cmab/ Pmab, were comparable. The ORR, DCR, and AEs grade $\geq 3$ were not significantly different between all the irinotecan-based and oxaliplatin-based treatments (Supplementary Tables 4-6).

Compared with the common regimens, namely, FOLFOX, FOLFIRI, and FOLFOXIRI, the addition of Bmab and/or Cmab and/or Pmab to these regimens resulted in a significant improvement in some treatment outcomes. Compared with FOLFIRI, FOLFIRI + $\mathrm{Bmab} / \mathrm{Cmab} / \mathrm{Pmab}$ increased the ORR, FOLFIRI + Bmab increased the DCR, and FOLFIRI + Cmab and FOLFIRI + Bmab + Pmab prolonged both OS and PFS, whereas FOLFIRI + Bmab prolonged PFS only, but safety outcomes were not significantly different. In addition, FOLFOX + Pmab was associated with a greater number of SAEs than FOLFOX. The ORR and the DCR of the FOLFOXIRI + Bmab group were better than those of the FOLFIRI group, and FOLFOXIRI + Pmab exerted better effects on the ORR and the DCR than did FOLFIRI, FOLFOX, and FOLFOXIRI (Supplementary Tables 4-6). The greater the number of drugs in the combination regimen, the higher the potential efficacy; however, the safety profile might remain similar.

Ba-Sang et al. reported the comparative response of patients to 17 regimens using an NMA and suggested the potential effect of Bmab + chemotherapy and Pmab + chemotherapy regimens compared with other targeted therapies (6). Another NMA of 10 regimens recommended considering FOLFIRI and FOLFOX as 

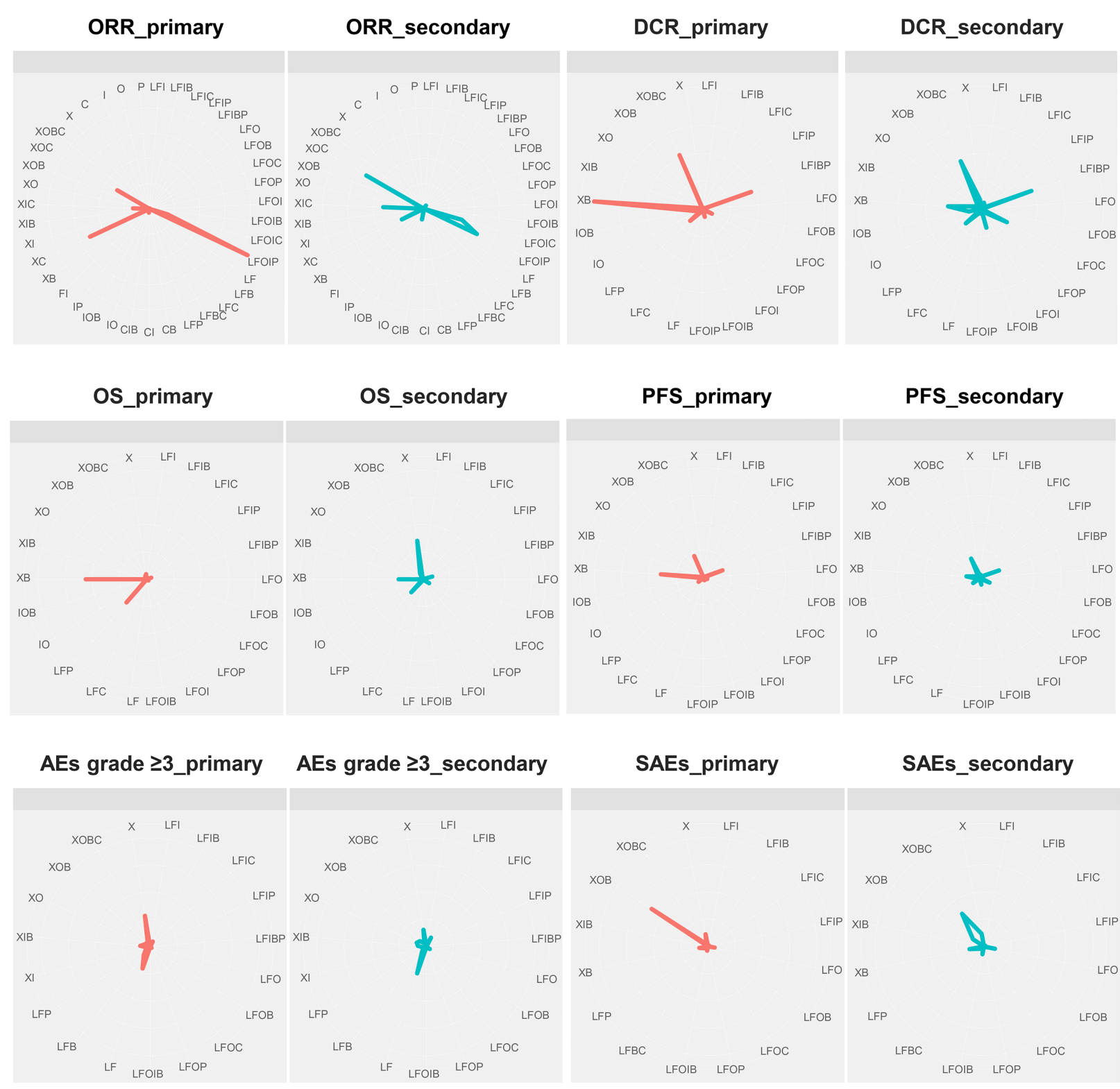

FIGURE 7 | First-line and second-line treatment ranking probabilities. ORR, overall response rate; DCR, disease control rate; OS, overall survival; PFS, progression-free survival; AEs, adverse events; SAEs, serious adverse events; X, capecitabine; B, bevacizumab; C, cetuximab; F, 5-fluorouracil; I, irinotecan; L, leucovorin; O, oxaliplatin; P, panitumumab. 
TABLE 2 | SUCRA rankings for colorectal cancer treatments.

\begin{tabular}{|c|c|c|c|c|c|c|}
\hline Treatment & ORR & DCR & os & PFS & AEs grade $\geq 3$ & SAEs \\
\hline LFI & 0.34 & 0.42 & 0.41 & 0.42 & 0.27 & 0.20 \\
\hline LFIB & 0.57 & 0.89 & 0.51 & 0.46 & 0.35 & 0.23 \\
\hline LFIC & 0.67 & 0.49 & 0.57 & 0.49 & 0.41 & 0.37 \\
\hline LFIP & 0.85 & 0.59 & 0.61 & 0.54 & 0.48 & 0.42 \\
\hline LFIBP & 0.67 & 0.76 & 0.62 & 0.54 & 0.52 & \\
\hline LFO & 0.49 & 0.59 & 0.61 & 0.51 & 0.56 & 0.48 \\
\hline LFOB & 0.59 & 0.85 & 0.59 & 0.49 & 0.57 & 0.52 \\
\hline LFOC & 0.82 & 0.70 & 0.57 & 0.48 & 0.58 & 0.55 \\
\hline LFOP & 0.68 & 0.72 & 0.55 & 0.49 & 0.59 & 0.57 \\
\hline LFOI & 0.60 & 0.70 & 0.52 & 0.50 & & \\
\hline LFOIB & 0.82 & 0.93 & 0.50 & 0.52 & 0.59 & 0.59 \\
\hline LFOIC & 0.88 & & & & & \\
\hline LFOIP & 0.96 & 0.99 & & 0.53 & & \\
\hline LF & 0.08 & 0.26 & 0.49 & 0.54 & 0.58 & \\
\hline LFB & 0.31 & 0.45 & & & 0.56 & \\
\hline LFC & 0.17 & & 0.50 & 0.54 & & \\
\hline LFBC & 0.35 & 0.70 & & & & 0.59 \\
\hline LFP & 0.63 & & 0.51 & 0.55 & 0.54 & 0.59 \\
\hline CB & 0.31 & & & & & \\
\hline $\mathrm{Cl}$ & 0.66 & 0.38 & & & & \\
\hline $\mathrm{CIB}$ & 0.74 & 0.45 & & & & \\
\hline 10 & 0.39 & 0.36 & 0.51 & 0.55 & & \\
\hline IOB & 0.46 & 0.27 & 0.51 & 0.55 & & \\
\hline $\mathrm{IP}$ & 0.57 & 0.23 & & & & \\
\hline $\mathrm{Fl}$ & 0.27 & 0.36 & & & & \\
\hline$X B$ & 0.24 & 0.41 & 0.51 & 0.54 & & 0.59 \\
\hline$x C$ & 0.73 & 0.36 & & & & \\
\hline$X I$ & 0.40 & 0.59 & & & 0.53 & \\
\hline XIB & 0.49 & 0.73 & 0.51 & 0.53 & 0.52 & 0.59 \\
\hline $\mathrm{XIC}$ & 0.88 & 0.35 & & & & \\
\hline xO & 0.36 & 0.53 & 0.48 & 0.51 & 0.51 & \\
\hline $\mathrm{XOB}$ & 0.43 & 0.55 & 0.38 & 0.47 & 0.49 & 0.58 \\
\hline XOC & 0.93 & 0.61 & & & & \\
\hline $\mathrm{XOBC}$ & 0.48 & 0.54 & 0.26 & 0.43 & 0.46 & 0.57 \\
\hline X & 0.09 & 0.18 & 0.30 & 0.35 & & 0.55 \\
\hline C & 0.20 & 0.11 & & & & \\
\hline 1 & 0.09 & 0.13 & & & & \\
\hline 0 & 0.06 & 0.18 & & & 0.40 & \\
\hline$P$ & 0.26 & 0.14 & & & & \\
\hline
\end{tabular}

ORR, overall response rate; DCR, disease control rate; OS, overall survival; PFS, progression-free survival; AEs, adverse events; SAEs, serious adverse events; $X$, capecitabine; $B$, bevacizumab; C, cetuximab; F, 5-fluorouracil; I, irinotecan; L, leucovorin; O, oxaliplatin; P, panitumumab.

first-line therapies for short-term and long-term advanced CRC treatments (7). According to the US Food and Drug Administration, FOLFOX is indicated for the treatment of adjuvant stage III or advanced CRC, whereas FOLFIRI is approved as a first-line treatment for metastatic CRC (119, 120). However, in the current NMA, the prolongation of OS and PFS by FOLFOX relative to FOLFIRI suggests a possible indication of FOLFOX for metastatic CRC. Furthermore, by implementing different approaches and selecting regimens using the NMA, the current study focused on the commonly used drugs in clinical practice and suggested that not only FOLFIRI and FOLFOX but also their combination with Bmab or Cmab or Pmab into these regimens might result in superior efficacy or safety. Consistent with our findings, Bmab, which is a vascular endothelial growth factor (VEGF) monoclonal antibody, is also indicated as a second-line treatment for metastatic CRC in combination with $5-\mathrm{FU}+$ irinotecan-based or 5-FU + oxaliplatin-based chemotherapy (121). Other epidermal growth factor receptor (EGFR) inhibitors, namely, Cmab and Pmab, can be administered as single agents after the failure of irinotecanbased and oxaliplatin-based regimens (122, 123). Similar findings were observed upon comparing the ORR and the DCR of Cmab or Pmab vs FOLFIRI or FOLFOX, Cmab vs. FOLFIRI + Cmab or FOLFOX + Cmab, and Pmab vs. FOLFIRI + Pmab or FOLFOX + Pmab regimens. Although data for survival and safety outcomes of patients treated with Cmab or Pmab are available, they do not contribute to the connection to the main network; thus, these treatment effects were not evaluated.

Given the systemic toxicity and unpredictable resistance to chemotherapy, Xie et al. recently conducted a comprehensive review of targeted therapy for patients with CRC (124). Most currently approved agents for patients with advanced-stage metastatic disease target EGFR- and VEGF-related pathways, namely, Cmab and Pmab as EGFR inhibitors, vemurafenib as a BRAF inhibitor, dabrafenib + trametinib and encorafenib + binimetinib as MEK inhibitors, and Bmab, regorafenib, ziv- 

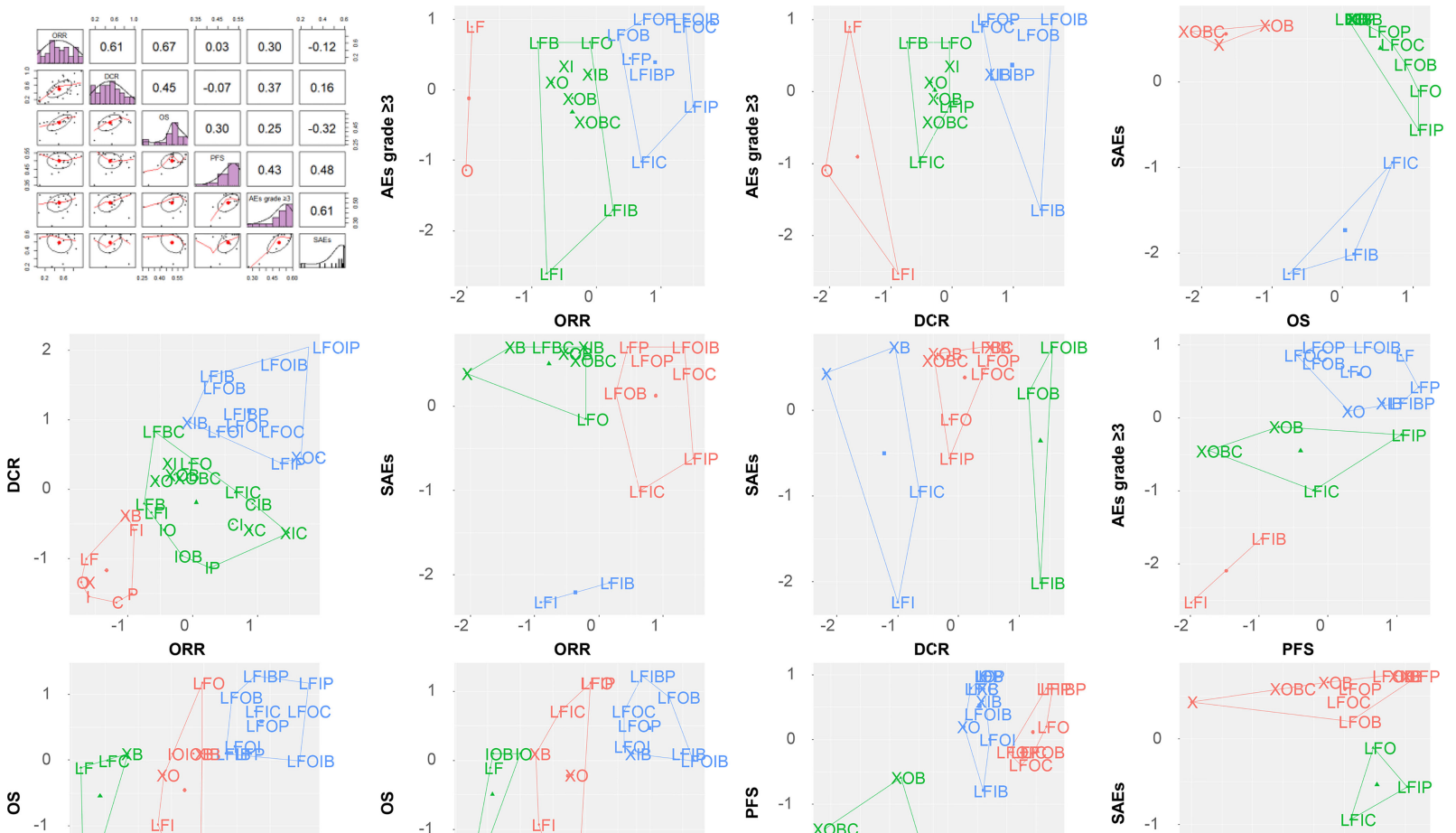

$$
\begin{aligned}
& \text { LFO LFIBP LFIP } \\
& \text { LFOB LFIC LFOC }
\end{aligned}
$$
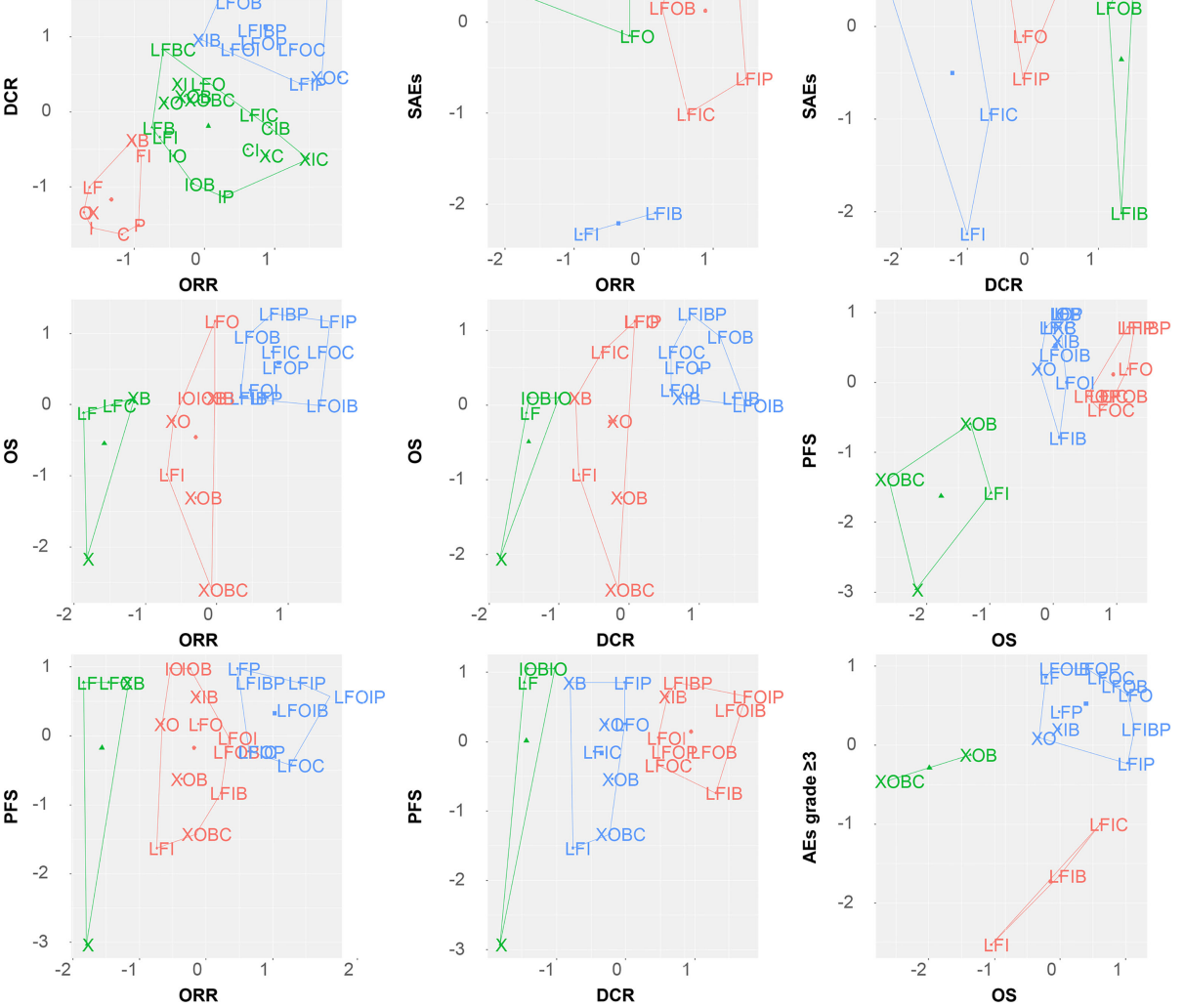

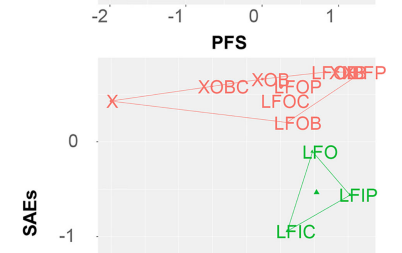

$-2$
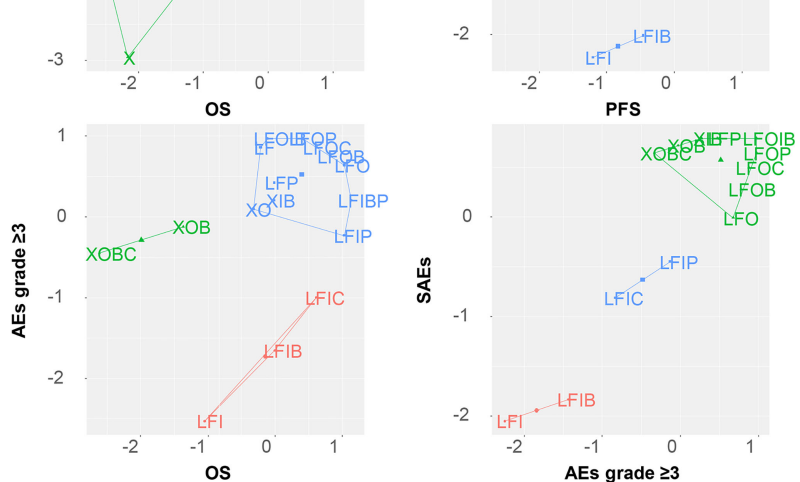

FIGURE 8 | SUCRA ranking plot and cluster analysis. ORR, overall response rate; DCR, disease control rate; OS, overall survival; PFS, progression-free survival; AEs, adverse events; SAEs, serious adverse events; X, capecitabine; B, bevacizumab; C, cetuximab; F, 5-fluorouracil; I, irinotecan; L, leucovorin; O, oxaliplatin; P, panitumumab.

aflibercept, and ramucirumab as treatments targeting angiogenesis (124). Among them, anti-EGFR agents are recommended for patients with left-side metastatic CRC with the wild-type RAS genotype only, whereas anti-VEGF agents can be used regardless of the RAS mutation status (124). Furthermore, a 10\% of BRAF (with common V600E substitution) mutation rate was reported in metastatic CRC (125). For these BRAF-mutated patients, chemotherapy + Bmab and FOLFOXIRI + Bmab showed good performance as a first-line treatment (125). However, regardless of the side and mutation status, our study determined the high efficacy of FOLFOXIRI + Pmab in terms of response and FOLFIRI + Bmab + Pmab in terms of survival outcomes. We also reported FOLFOXIRI + Bmab as a treatment with high safety.
To the knowledge of the authors, this study is the first to investigate both the efficacy and safety of common drugs used to treat advanced or metastatic CRC. The individual studies were derived from a large number of RCTs and participants. Efficacy and safety were investigated through different surrogates to yield consistent findings. The statistical analysis was conducted using the Bayesian approach, with the appropriate prior distribution of variables, which produced reliable results. Bayesian assumptions, such as consistency and heterogeneity, were checked and reported. A sensitivity analysis among three core models was also performed to obtain robust estimates.

Despite these advantages, the current NMA has some limitations. First, due to a lack of head-to-head trials, several 
treatments were excluded from the network of different outcomes. Second, the combination of treatments with different dosage forms and the treatment plan might have affected the final estimates. Third, the limitation of the meta-analysis in considering differences across studies due to patient characteristics must be addressed. Recent methods have been proposed to account the heterogeneity between trial results in meta-analyses $(126,127)$; however, the application of NMA requires further investigation. Fourth, the current study did not yield an ideal index for both efficacy and safety from six evaluated endpoints to select the ideal regimen for the treatment of advanced or metastatic CRC. Additional research data and an optimized algorithm are required to correct this deviation $(128,129)$.

In summary, we performed an NMA of available RCTs to investigate the efficacy and safety of various therapies for advanced or metastatic CRC. We observed good efficacy of FOLFOXIRI + Pmab in terms of response and FOLFIRI + $\mathrm{Bmab}+\mathrm{Pmab}$ in terms of survival outcomes and good safety profile for FOLFOXIRI + Bmab. We propose that this overview provides the most appropriate evidence for a range of efficient therapies and may inform clinical practice and decision making and aid in the planning for future research. Further studies may develop a novel endpoint that considers both the efficacy and safety of regimens and consider the side and mutation status to confirm our findings.

\section{DATA AVAILABILITY STATEMENT}

The original contributions presented in the study are included in the article/Supplementary Material. Further inquiries can be directed to the corresponding author.

\section{REFERENCES}

1. Bray F, Ferlay J, Soerjomataram I, Siegel RL, Torre LA, Jemal A. Global Cancer Statistics 2018: GLOBOCAN Estimates of Incidence and Mortality Worldwide for 36 Cancers in 185 Countries. CA Cancer J Clin (2018) 68:394-4245. doi: 10.3322/caac. 21492

2. Cunningham D, Atkin W, Lenz HJ, Lynch HT, Minsky B, Nordlinger B, et al. Colorectal Cancer. Lancet (2010) 375:1030-475. doi: 10.1016/S01406736(10)60353-4

3. NCCN. Colon Cancer. Clinical Practice Guidelines in Oncology (Version 2.2016. 2015) (2017). Available at: https://www.nccn.org/professionals/ physician_gls/pdf/colon.pdf.

4. Joseph TD, Gary CY, Michael LP. Pharmacotherapy: A Pathophisiologic Approach, 11th. McGraw-Hill (2020).

5. Rouse B, Chaimani A, Li T. Network Meta-Analysis: An Introduction for Clinicians. Intern Emerg Med (2017) 12:103-15. doi: 10.1007/s11739-016-1583-7

6. Ba-Sang DZ, Long ZW, Teng H, Zhao XP, Qiu J, Li MS. A Network MetaAnalysis on the Efficacy of Sixteen Targeted Drugs in Combination With Chemotherapy for Treatment of Advanced/Metastatic Colorectal Cancer. Oncotarget (2016) 7:84468-795. doi: 10.18632/oncotarget.12994

7. Wu DM, Wang YJ, Fan SH, Zhuang J, Zhang ZF, Shan Q, et al. Network Meta-Analysis of the Efficacy of First-Line Chemotherapy Regimens in Patients With Advanced Colorectal Cancer. Oncotarget (2017) 8:100668775. doi: 10.18632/oncotarget.22177

8. Liberati A, Altman DG, Tetzlaff J, Mulrow C, Gotzsche PC, Ioannidis JP, et al. The PRISMA Statement for Reporting Systematic Reviews and Meta-

\section{AUTHOR CONTRIBUTIONS}

We greatly appreciate the efforts of those individuals who contributed to this study. The authors' responsibilities were as follows: TH and JK designed the research. TH, DS, BK, YC, and JK conducted the research. TH analyzed the data. TH and JK wrote the paper and are primarily responsible for the final content. All authors contributed to the article and approved the submitted version.

\section{FUNDING}

This work was supported by grants from the National Cancer Center (Nos. 1710882 and 2210990).

\section{ACKNOWLEDGMENTS}

The author, TH, expresses appreciation for the support from the "Global Leadership" program of the Korea Safety Health Environment Foundation. We thank Tho Thi Anh Tran, from Nghe An Oncology Hospital, for her useful comments on this study.

\section{SUPPLEMENTARY MATERIAL}

The Supplementary Material for this article can be found online at: https://www.frontiersin.org/articles/10.3389/fonc.2021.756214/ full\#supplementary-material

Analyses of Studies That Evaluate Healthcare Interventions: Explanation and Elaboration. BMJ (2009) 339:b2700. doi: 10.1136/bmj.b2700

9. Higgins JP, Altman DG, Gotzsche PC, Juni P, Moher D, Oxman AD, et al. The Cochrane Collaboration's Tool for Assessing Risk of Bias in Randomised Trials. BMJ (2011) 343:d5928. doi: 10.1136/bmj.d5928

10. van Valkenhoef G, Kuiper J. Package 'Gemtc': Network Meta-Analysis Using Bayesian Methods (Version 1.0-1) (2021). Available at: https://cran.r-project. org/web/packages/gemtc/gemtc.pdf.

11. Dias S, Sutton AJ, Ades AE, Welton NJ. Evidence Synthesis for Decision Making 2: A Generalized Linear Modeling Framework for Pairwise and Network Meta-Analysis of Randomized Controlled Trials. Med Decis Making (2013) 33:607-175. doi: 10.1177/0272989X12458724

12. Dias S, Sutton AJ, Welton NJ, Ades AE. Evidence Synthesis for Decision Making 3: Heterogeneity-subgroups, Meta-Regression, Bias, and BiasAdjustment. Med Decis Making (2013) 33:618-405. doi: 10.1177/ 0272989X13485157

13. Chaimani A, Higgins JP, Mavridis D, Spyridonos P, Salanti G. Graphical Tools for Network Meta-Analysis in Stata. PloS One (2013) 8:e766545. doi: 10.1371/journal.pone.0076654

14. Mbuagbaw L, Rochwerg B, Jaeschke R, Heels-Andsell D, Alhazzani W, Thabane L, et al. Approaches to Interpreting and Choosing the Best Treatments in Network Meta-Analyses. Syst Rev (2017) 6:795. doi: 10.1186/s13643-017-0473-z

15. Revelle W. Package 'Psych': Procedures for Psychological, Psychometric, and Personality Research (Version 2.1.6) (2021). Available at: https://cran.r-project. org/web/packages/psych/psych.pdf [Accessed date: December 12, 2020]. 
16. Kassambara A, Mundt F. Package 'Factoextra': Extract and Visualize the Results of Multivariate Data Analyses (Version 1.0.7) (2020). Available at: https://cloud.r-project.org/web/packages/factoextra/factoextra.pdf. [Accessed date: December 12, 2020]

17. Lonardi S, Schirripa M, Buggin F, Antonuzzo L, Merelli B, Boscolo G, et al. First-Line FOLFOX Plus Panitumumab Versus 5FU Plus Panitumumab in RAS-BRAF Wildtype Metastatic Colorectal Cancer Elderly Patients: The Panda Study. J Clin Oncol (2020) 38:4002. doi: 10.1200/JCO.2020.38.15_suppl.4002

18. Lipsyc-Sharf M, Ou FS, Yurgelun MB, Rubinson DA, Schrag D, Dakhil SR, et al. Irinotecan, Cetuximab, and Bevacizumab (CBI) Versus Irinotecan, Cetuximab, and Placebo (CI) in Irinotecanrefractory Metastatic Colorectal Cancer (mCRC): Results From an ACCRU Network Randomized Phase II Trial. J Clin Oncol (2020) 38:102. doi: 10.1200/JCO.2020.38.4_suppl.102

19. Hu H, Wang K, Wang W, Qiu M, Lin R, Zhang H, et al. ACCRU With or Without Cetuximab as Conversion Therapy in Patients With Ras/BRAF Wildtype Unresectable Liver Metastases Colorectal Cancer: The FOCULM Study. J Clin Oncol (2020) 38:99. doi: 10.1200/JCO.2020.38.4_suppl.99

20. Guo W, Zhang X, Wang Y, Zhang W, Liu X, Shen W, et al. FOLFIRI Versus Irinotecan Monodrug as Second-Line Treatment in Metastatic Colorectal Cancer Patients: An Open, Multicenter, Prospective, Randomized Controlled Phase III Clinical Study. J Clin Oncol (2020) 38:4038. doi: 10.1200/JCO.2020.38.15_suppl.4038

21. Folprecht G, Mende M, Liersch T, Bechstein WO, Kohne CH, Stein A, et al. Cetuximab/irinotecan/5-FU +/-Oxaliplatin or FOLFOXIRI +/-Bevacizumab in Patients With Colorectal Cancer and Nonresectable Liver Metastases (AIO CELIM2-Study). J Clin Oncol (2020) 38:4024. doi: 10.1200/ JCO.2020.38.15_suppl.4024

22. Xu J, Liu T, Tang W, Chang W, Feng Q, Wei Y, et al. Bevacizumab Plus Chemotherapy Versus Chemotherapy Alone as First-Line Treatment for Patients With RAS Mutant Unresectable Colorectal Liver-Limited Metastases: A Single Center Randomized Control Trial. Ann Oncol (2019) 30:v867. doi: 10.1093/annonc/mdz394.020

23. Pietrantonio F, Morano F, Corallo S, Miceli R, Lonardi S, Raimondi A, et al. Maintenance Therapy With Panitumumab Alone vs Panitumumab Plus Fluorouracil-Leucovorin in Patients With RAS Wild-Type Metastatic Colorectal Cancer: A Phase 2 Randomized Clinical Trial. JAMA Oncol (2019) 5(9):1268-75. doi: 10.1001/jamaoncol.2019.1467

24. Kienle DL, Dietrich D, Ribi K, Wicki A, Quagliata L, Winterhalder RC, et al. Cetuximab Monotherapy and Cetuximab Plus Capecitabine as First-Line Treatment in Older Patients With RAS- and BRAF Wild-Type Metastatic Colorectal Cancer. Results of the Multicenter Phase II Trial SAKK 41/10. J Geriatr Oncol (2019) 10:304-10. doi: 10.1016/j.jgo.2018.11.011

25. Uetake H, Emi Y, Yamanaka T, Muro K, Oki E, Takahashi T, et al. A Randomized Phase II Study of mFOLFOX6 Plus Bevacizumab Versus mFOLFOX6 Plus Cetuximab for Previously Untreated, Liverlimited Metastatic Colorectal Cancer That Is Unsuitable for Resection (ATOM Trial). J Clin Oncol (2018) 36:734. doi: 10.1200/JCO.2018.36.4-suppl.734

26. Shapiro JD, Thavaneswaran S, Underhill CR, Robledo KP, Karapetis CS, Day FL, et al. Cetuximab Alone or With Irinotecan for Resistant KRAS-, NRAS-, BRAF- and PIK3CA-Wild-Type Metastatic Colorectal Cancer: The Agitg Randomized Phase II ICECREAM Study. Clin Colorectal Cancer (2018) 17:313-195. doi: 10.1016/j.clcc.2018.06.002

27. Qin S, Guo W, Xu JM, Li Q, Cheng Y, Liu TS, et al. Final Overall Survival (OS) Analysis of First-Line (1L) FOLFOX-4 \pm Cetuximab (Cet) in Patients (Pts) With RAS Wild-Type (Wt) Metastatic Colorectal Cancer (mCRC) in the Phase 3 TAILOR Trial. J Clin Oncol (2018) 36:3521. doi: 10.1200/ JCO.2018.36.15_suppl.3521

28. Nakayama G, Mitsuma A, Sunagawa $Y$, Ishigure K, Yokoyama H, Matsui T, et al. Randomized Phase II Trial of CapOX Plus Bevacizumab and CapIRI Plus Bevacizumab as First-Line Treatment for Japanese Patients With Metastatic Colorectal Cancer (CapIRI-1201 Study). Oncologist (2018) 23:919-275. doi: 10.1634/theoncologist.2017-0640

29. Meropol NJ, Feng Y, Grem JL, Mulcahy MF, Catalano PJ, Kauh JS, et al. Phase 2 Study of Treatment Selection Based on Tumor Thymidylate Synthase Expression in Previously Untreated Patients With Metastatic Colorectal Cancer: A Trial of the CapIRI Cancer Research Group (E4203). Cancer (2018) 124:688-975. doi: 10.1002/cncr.30967
30. Maiello E, Di Maggio G, Cordio SS, Cinieri S, Giuliani F, Pisconti S, et al. Bevacizumab (B) + Bi-Weekly Capecitabine (C) and Oxaliplatin (O) (XELOX2) or FOLFOX4 in First-Line Treatment of Metastatic Colorectal Cancer (mCRC): Final Results of a Multicenter Randomized Phase II Trial of the Gruppo Oncologico Dell'italia Meridionale (GOIM Protocol 2802). J Clin Oncol (2018) 36:3542. doi: 10.1200/JCO.2018.36.15_suppl.3542

31. Hurwitz HI, Tan BR, Reeves JA, Xiong H, Somer B, Lenz HJ, et al. Phase II Randomized Trial of Sequential or Concurrent FOLFOXIRI-Bevacizumab Versus FOLFOX-Bevacizumab for Metastatic Colorectal Cancer (Steam). Oncologist (2018) 24(7):921-32. doi: 10.1634/theoncologist.2018-0344

32. Hou G, Song R, Yang J, Zhang Y, Xiao C, Wang C, et al. Treatment Effect of Conversion Therapy and Its Correlation With VEGF Expression in Unresectable Rectal Cancer With Liver Metastasis. Oncol Lett (2018) 16:749-54. doi: 10.3892/ol.2018.8758

33. Gomez A, Vieitez JM, Gil S, Salvia AS, Suárez BG, Alfonso PG, et al. Safety Analysis of a Phase III Randomized Trial Comparing FOLFOX + Bevacizumab vs FOLFOXIRI + Bevacizumab as 1st Line Treatment in Patients With Metastatic Colorectal Cancer (mCRC) With $\geq 3$ Circulating Tumor Cells (CTCs) (VISNU-1 TTD TRIAL). J Clin Oncol (2018) 36:3536. doi: 10.1200/JCO.2018.36.15_suppl.3536

34. Geissler M, Klingler T, Riera-Knorrenschield J, Tannapfel A, Seufferlein T, Held S, et al. MFOLFOXIRI 1 Panitumumab Versus FOLFOXIRI as FirstLine Treatment in Patients With RAS Wild- Type Metastatic Colorectal Cancer $\mathrm{m}(\mathrm{CRC})$ : A Randomized Phase II VOLFI Trial of the AIO (AIOKRK0109). Ann Oncol (2018) 29:v108. doi: 10.1093/annonc/mdy149

35. Sugimoto N, Sakai D, Tamura T, Hara H, Nishina T, Esaki T, et al. Randomized Phase II Study of Panitumumab (Pmab) + Irinotecan (CPT11) Versus Cetuximab (Cmab) + CPT-11 in Patients (Pts) With KRAS WildType (WT) Metastatic Colorectal Cancer (mCRC) After Fluoropyrimidine (FU), CPT-11, and Oxaliplatin (L-OHP) Failure: WJOG6510G. J Clin Oncol (2017) 35:661. doi: 10.1200/JCO.2017.35.4_suppl.661

36. Schmoll HJ, Meinert FM, Cygon F, Garlipp B, Junghanss C, Leithäuser M, et al. "CHARTA: FOLFOX/bevacizumab vs. FOLFOXIRI/bevacizumab in Advanced Colorectal Cancer-Final Results, Prognostic, and Potentially Predictive Factors From the Randomized Phase II Trial of the AIO. J Clin Oncol (2017) 35:3533. doi: 10.1200/JCO.2017.35.15_suppl.3533

37. Carrato A, Abad A, Massuti B, Gravalos C, Escudero P, Longo-Munoz F, et al. First-Line Panitumumab Plus FOLFOX4 or FOLFIRI in Colorectal Cancer With Multiple or Unresectable Liver Metastases: A Randomised, Phase II Trial (PLANET-TTD). Eur J Cancer (2017) 81:191-2025. doi: 10.1016/j.ejca.2017.04.024

38. Yamazaki K, Nagase M, Tamagawa H, Ueda S, Tamura T, Murata K, et al. Randomized Phase III Study of Bevacizumab Plus FOLFIRI and Bevacizumab Plus mFOLFOX6 as First-Line Treatment for Patients With Metastatic Colorectal Cancer (WJOG4407G). Ann Oncol (2016) 27:1539465. doi: 10.1093/annonc/mdw206

39. Shitara K, Yonesaka K, Denda T, Yamazaki K, Moriwaki T, Tsuda M, et al. Randomized Study of FOLFIRI Plus Either Panitumumab or Bevacizumab for Wild-Type KRAS Colorectal Cancer-Wjog 6210g. Cancer Sci (2016) 107:1843-505. doi: 10.1111/cas.13098

40. Segelov E, Thavaneswaran S, Waring PM, Desai J, Robledo KP, Gebski VJ, et al. Response to Cetuximab With or Without Irinotecan in Patients With Refractory Metastatic Colorectal Cancer Harboring the KRAS G13D Mutation: Australasian Gastro-Intestinal Trials Group ICECREAM Study. J Clin Oncol (2016) 34:2258-645. doi: 10.1200/jco.2015.65.6843

41. Ciardiello F, Normanno N, Martinelli E, Troiani T, Pisconti S, Cardone C, et al. Cetuximab Continuation After First Progression in Metastatic Colorectal Cancer (CAPRI-GOIM): A Randomized Phase II Trial of FOLFOX Plus Cetuximab Versus FOLFOX. Ann Oncol (2016) 27:1055615. doi: 10.1093/annonc/mdw136

42. Aparicio T, Lavau-Denes S, Phelip JM, Maillard E, Jouve JL, Gargot D, et al. Randomized Phase III Trial in Elderly Patients Comparing LV5FU2 With or Without Irinotecan for First-Line Treatment of Metastatic Colorectal Cancer (FFCD 2001-02). Ann Oncol (2016) 27:121-75. doi: 10.1093/annonc/mdv491

43. Tournigand C, Chibaudel B, Samson B, Scheithauer W, Lledo G, Artru P, et al. Improving Safety in First-Line Metastatic Colorectal Cancer (mCRC) Therapy With Bevacizumab: Modified FOLFOX7 Versus XELOX2-Results 
of the Induction Phase of the GERCOR DREAM Randomized Phase III Study. J Clin Oncol (2015) 33:670. doi: 10.1200/jco.2015.33.3_suppl.670

44. Passardi A, Nanni O, Tassinari D, Turci D, Cavanna L, Fontana A, et al. Effectiveness of Bevacizumab Added to Standard Chemotherapy in Metastatic Colorectal Cancer: Final Results for First-Line Treatment From the ITACa Randomized Clinical Trial. Ann Oncol (2015) 26:1201-75. doi: 10.1093/annonc/mdv130

45. Liu Y, Luan L, Wang X. Arandomized Phase II Clinical Study of Combining Panitumumab and Bevacizumab, Plus Irinotecan, 5-Fluorouracil, and Leucovorin (FOLFIRI) Compared With FOLFIRI Alone as Second-Line Treatment for Patients With Metastatic Colorectal Cancer and KRAS Mutation. Onco Targets Ther (2015) 8:1061-685. doi: 10.2147/OTT.S81442

46. Hecht JR, Cohn A, Dakhil S, Saleh M, Piperdi B, Cline-Burkhardt M, et al. SPIRITT: A Randomized, Multicenter, Phase II Study of Panitumumab With FOLFIRI and Bevacizumab With FOLFIRI as Second-Line Treatment in Patients With Unresectable Wild Type KRAS Metastatic Colorectal Cancer. Clin Colorectal Cancer (2015) 14:72-805. doi: 10.1016/j.clcc.2014.12.009

47. Gruenberger T, Bridgewater J, Chau I, Garcia Alfonso P, Rivoire M, Mudan S, et al. Bevacizumab Plus mFOLFOX-6 or FOLFIRI in Patients With Initially Unresectable Liver Metastases From Colorectal Cancer: The OLIVIA Multinational Randomised Phase II Trial. Ann Oncol (2015) 26:702-85. doi: 10.1093/annonc/mdu580

48. Cremolini C, Loupakis F, Antoniotti C, Lupi C, Sensi E, Lonardi S, et al. FOLFOXIRI Plus Bevacizumab Versus FOLFIRI Plus Bevacizumab as First-Line Treatment of Patients With Metastatic Colorectal Cancer: Updated Overall Survival and Molecular Subgroup Analyses of the Open-Label, Phase 3 TRIBE Study. Lancet Oncol (2015) 16:1306-155. doi: 10.1016/s1470-2045(15)00122-9

49. Xie S, Han G, Fan Z, He L, Xu W, Qin Z. Safety and Efficacy of Second-Line Treatment With Folinic Acid, 5-Fluorouracil and Irinotecan (FOLFIRI) in Combination of Panitumumab and Bevacizumab for Patients With Metastatic Colorectal Cancer. Med Oncol (2014) 31:355. doi: 10.1007/s12032-014-0035-3

50. Schwartzberg LS, Rivera F, Karthaus M, Fasola G, Canon JL, Hecht JR, et al. Peak: A Randomized, Multicenter Phase II Study of Panitumumab Plus Modified Fluorouracil, Leucovorin, and Oxaliplatin (mFOLFOX6) or Bevacizumab Plus mFOLFOX6 in Patients With Previously Untreated, Unresectable, Wild-Type KRAS Exon 2 Metastatic Colorectal Cancer. J Clin Oncol (2014) 32:2240-75. doi: 10.1200/jco.2013.53.2473

51. Price TJ, Peeters M, Kim TW, Li J, Cascinu S, Ruff P, et al. Panitumumab Versus Cetuximab in Patients With Chemotherapy-Refractory Wild-Type KRAS Exon 2 Metastatic Colorectal Cancer (ASPECCT): A Randomised, Multicentre, Open-Label, Non-Inferiority Phase 3 Study. Lancet Oncol (2014) 15:569-795. doi: 10.1016/s1470-2045(14)70118-4

52. Peeters M, Price TJ, Cervantes A, Sobrero AF, Ducreux M, Hotko Y, et al. Final Results From a Randomized Phase 3 Study of FOLFIRI \pm Panitumumab for Second-Line Treatment of Metastatic Colorectal Cancer. Ann Oncol (2014) 25:107-65. doi: 10.1093/annonc/mdt523

53. Heinemann V, von Weikersthal LF, Decker T, Kiani A, Vehling-Kaiser U, Al-Batran SE, et al. FOLFIRI Plus Cetuximab Versus FOLFIRI Plus Bevacizumab as First-Line Treatment for Patients With Metastatic Colorectal Cancer (Fire-3): A Randomised, Open-Label, Phase 3 Trial. Lancet Oncol (2014) 15:1065-755. doi: 10.1016/s1470-2045(14)70330-4

54. Folprecht G, Gruenberger T, Bechstein W, Raab HR, Weitz J, Lordick F, et al. Survival of Patients With Initially Unresectable Colorectal Liver Metastases Treated With FOLFOX/Cetuximab or FOLFIRI/Cetuximab in a Multidisciplinary Concept (CELIM Study). Ann Oncol (2014) 25:1018255. doi: 10.1093/annonc/mdu088

55. Douillard JY, Siena S, Cassidy J, Tabernero J, Burkes R, Barugel M, et al. Final Results From PRIME: Randomized Phase III Study of Panitumumab With FOLFOX4 for First-Line Treatment of Metastatic Colorectal Cancer. Ann Oncol (2014) 25:1346-555. doi: 10.1093/annonc/mdu141

56. Cao R, Zhang S, Ma D, Hu L. A Multi-Center Randomized Phase II Clinical Study of Bevacizumab Plus Irinotecan, 5-Fluorouracil, and Leucovorin (FOLFIRI) Compared With FOLFIRI Alone as Second-Line Treatment for Chinese Patients With Metastatic Colorectal Cancer. Med Oncol (2014) 32:1-55. doi: 10.1007/s12032-014-0325-9

57. Seymour MT, Brown SR, Middleton G, Maughan T, Richman S, Gwyther S, et al. Panitumumab and Irinotecan Versus Irinotecan Alone for Patients With KRAS Wild-Type, Fluorouracil-Resistant Advanced Colorectal Cancer
(PICCOLO): A Prospectively Stratified Randomised Trial. Lancet Oncol (2013) 14:749-595. doi: 10.1016/s1470-2045(13)70163-3

58. Schmiegel W, Reinacher-Schick A, Arnold D, Kubicka S, Freier W, Dietrich G, et al. Capecitabine/irinotecan or Capecitabine/Oxaliplatin in Combination With Bevacizumab Is Effective and Safe as First-Line Therapy for Metastatic Colorectal Cancer: A Randomized Phase II Study of the AIO Colorectal Study Group. Ann Oncol (2013) 24:1580-75. doi: 10.1093/annonc/mdt028

59. Personeni N, Rimassa L, Verusio C, Barni S, Destro A, Raschioni C, et al. Prognostic Factors in KRAS Wild-Type (Wt) Metastatic Colorectal Cancer (mCRC) Patients (Pts) Treated With Biweekly Cetuximab (C) Plus Irinotecan, Fluorouracil, and Leucovorin (FOLFIRI): A Phase II Study. J Clin Oncol (2013) 31:e14611. doi: 10.1200/jco.2013.31.15_suppl.e14611

60. Hong YS, Jung KH, Kim HJ, Kim KP, Kim SY, Lee JL, et al. Randomized Phase II Study of Capecitabine With or Without Oxaliplatin as First-Line Treatment for Elderly or Fragile Patients With Metastatic Colorectal Cancer: A Prospective, Multicenter Trial of the Korean Cancer Study Group Co06-01. Am J Clin Oncol (2013) 36:565-715. doi: 10.1097/COC.0b013e31825d52d5

61. Ducreux M, Adenis A, Pignon JP, Francois E, Chauffert B, Ichante JL, et al. Efficacy and Safety of Bevacizumab-Based Combination Regimens in Patients With Previously Untreated Metastatic Colorectal Cancer: Final Results From a Randomised Phase II Study of Bevacizumab Plus 5Fluorouracil, Leucovorin Plus Irinotecan Versus Bevacizumab Plus Capecitabine Plus Irinotecan (FNCLCC ACCORD 13/0503 Study). Eur J Cancer (2013) 49:1236-455. doi: 10.1016/j.ejca.2012.12.011

62. Cunningham D, Lang I, Marcuello E, Lorusso V, Ocvirk J, Shin DB, et al. Bevacizumab Plus Capecitabine Versus Capecitabine Alone in Elderly Patients With Previously Untreated Metastatic Colorectal Cancer (AVEX): An Open-Label, Randomised Phase 3 Trial. Lancet Oncol (2013) 14:1077855. doi: 10.1016/s1470-2045(13)70154-2

63. Tveit KM, Guren T, Glimelius B, Pfeiffer P, Sorbye H, Pyrhonen S, et al. "Phase III Trial of Cetuximab With Continuous or Intermittent Fluorouracil, Leucovorin, and Oxaliplatin (Nordic FLOX) Versus FLOX Alone in First-Line Treatment of Metastatic Colorectal Cancer: The NORDIC-VII Study. J Clin Oncol (2012) 30:1755-625. doi: 10.1200/ jco.2011.38.0915

64. Souglakos J, Ziras N, Kakolyris S, Boukovinas I, Kentepozidis N, Makrantonakis P, et al. Randomised Phase-II Trial of CAPIRI (Capecitabine, Irinotecan) Plus Bevacizumab vs FOLFIRI (Folinic Acid, 5Fluorouracil, Irinotecan) Plus Bevacizumab as First-Line Treatment of Patients With Unresectable/Metastatic Colorectal Cancer (mCRC). Br J Cancer (2012) 106:453-95. doi: 10.1038/bjc.2011.594

65. Saltz L, Badarinath S, Dakhil S, Bienvenu B, Harker WG, Birchfield G, et al. Phase III Trial of Cetuximab, Bevacizumab, and 5-Fluorouracil/Leucovorin vs. FOLFOX-Bevacizumab in Colorectal Cancer. Clin Colorectal Cancer (2012) 11:101-11. doi: 10.1016/j.clcc.2011.05.006

66. Pectasides D, Papaxoinis G, Kalogeras KT, Eleftheraki AG, Xanthakis I, Makatsoris T, et al. XELIRI-Bevacizumab Versus FOLFIRI-Bevacizumab as First-Line Treatment in Patients With Metastatic Colorectal Cancer: A Hellenic Cooperative Oncology Group Phase III Trial With Collateral Biomarker Analysis. BMC Cancer (2012) 12:2715. doi: 10.1186/14712407-12-271

67. Dotan E, Meropol NJ, Burtness B, Denlinger CS, Lee J, Mintzer D, et al. A Phase II Study of Capecitabine, Oxaliplatin, and Cetuximab With or Without Bevacizumab as Frontline Therapy for Metastatic Colorectal Cancer. A Fox Chase Extramural Research Study. J Gastrointest Cancer (2012) 43:562-9. doi: 10.1007/s12029-012-9368-3

68. Van Cutsem E, Kohne CH, Lang I, Folprecht G, Nowacki MP, Cascinu S, et al. Cetuximab Plus Irinotecan, Fluorouracil, and Leucovorin as First-Line Treatment for Metastatic Colorectal Cancer: Updated Analysis of Overall Survival According to Tumor KRAS and BRAF Mutation Status. J Clin Oncol (2011) 29:2011-95. doi: 10.1200/jco.2010.33.5091

69. Moosmann N, Von Weikersthal LF, Vehling-Kaiser U, Stauch M, Hass HG, Dietzfelbinger $\mathrm{H}$, et al. Cetuximab Plus Capecitabine and Irinotecan Compared With Cetuximab Plus Capecitabine and Oxaliplatin as FirstLine Treatment for Patients With Metastatic Colorectal Cancer: AIO KRK0104 - A Randomized Trial of the German Aio Crc Study Group. J Clin Oncol (2011) 29:1050-585. doi: 10.1200/JCO.2010.31.1936 
70. Masi G, Vasile E, Loupakis F, Cupini S, Fornaro L, Baldi G, et al. Randomized Trial of Two Induction Chemotherapy Regimens in Metastatic Colorectal Cancer: An Updated Analysis. J Natl Cancer Inst (2011) 103:21-305. doi: 10.1093/jnci/djq456

71. Guan ZZ, Xu JM, Luo RC, Feng FY, Wang LW, Shen L, et al. Efficacy and Safety of Bevacizumab Plus Chemotherapy in Chinese Patients With Metastatic Colorectal Cancer: A Randomized Phase III ARTIST Trial. Chin J Cancer (2011) 30:682-95. doi: 10.5732/cjc.011.10188

72. Fischer von Weikersthal L, Schalhorn A, Stauch M, Quietzsch D, Maubach PA, Lambertz $\mathrm{H}$, et al. Phase III Trial of Irinotecan Plus Infusional 5Fluorouracil/Folinic Acid Versus Irinotecan Plus Oxaliplatin as First-Line Treatment of Advanced Colorectal Cancer. Eur J Cancer (2011) 47:206-145. doi: 10.1016/j.ejca.2010.09.022

73. Ducreux M, Bennouna J, Hebbar M, Ychou M, Lledo G, Conroy T, et al. Capecitabine Plus Oxaliplatin (XELOX) Versus 5-Fluorouracil/Leucovorin Plus Oxaliplatin (FOLFOX-6) as First-Line Treatment for Metastatic Colorectal Cancer. Int J Cancer (2011) 128:682-905. doi: 10.1002/ijc.25369

74. Cassidy J, Clarke S, Diaz-Rubio E, Scheithauer W, Figer A, Wong R, et al. XELOX vs FOLFOX-4 as First-Line Therapy for Metastatic Colorectal Cancer: NO16966 Updated Results. Br J Cancer (2011) 105:58-645. doi: 10.1038/bjc.2011.201

75. Vamvakas L, Athanasiadis A, Karampeazis A, Kakolyris S, Polyzos A, Kouroussis C, et al. Clinical Outcome of Elderly Patients With Metastatic Colorectal Cancer Treated With FOLFOXIRI Versus FOLFIRI: Subgroup Analysis of a Randomized Phase III Trial From the Hellenic Oncology Research Group (HORG). Crit Rev Oncol Hematol (2010) 76:61-705. doi: 10.1016/j.critrevonc.2009.08.003

76. Peeters M, Price TJ, Cervantes A, Sobrero AF, Ducreux M, Hotko Y, et al. Randomized Phase III Study of Panitumumab With Fluorouracil, Leucovorin, and Irinotecan (FOLFIRI) Compared With FOLFIRI Alone as Second-Line Treatment in Patients With Metastatic Colorectal Cancer. J Clin Oncol (2010) 28:4706-135. doi: 10.1200/jco.2009.27.6055

77. Ocvirk J, Brodowicz T, Wrba F, Ciuleanu TE, Kurteva G, Beslija S, et al. Cetuximab Plus FOLFOX6 or FOLFIRI in Metastatic Colorectal Cancer: Cecog Trial. World J Gastroenterol (2010) 16:3133-435. doi: 10.3748/wjg. v16.i25.3133

78. Folprecht G, Gruenberger T, Bechstein WO, Raab HR, Lordick F, Hartmann JT, et al. Tumour Response and Secondary Resectability of Colorectal Liver Metastases Following Neoadjuvant Chemotherapy With Cetuximab: The CELIM Randomised Phase 2 Trial. Lancet Oncol (2010) 11:38-475. doi: 10.1016/S1470-2045(09)70330-4

79. Tol J, Koopman M, Cats A, Rodenburg CJ, Creemers GJ, Schrama JG, et al. Chemotherapy, Bevacizumab, and Cetuximab in Metastatic Colorectal Cancer. N Engl J Med (2009) 360:563-725. doi: 10.1056/NEJMoa0808268

80. Moehler M, Sprinzl MF, Abdelfattah M, Schimanski CC, Adami B, Godderz W, et al. Capecitabine and Irinotecan With and Without Bevacizumab for Advanced Colorectal Cancer Patients. World J Gastroenterol (2009) 15:449565. doi: 10.3748/wjg.15.449

81. Bokemeyer C, Bondarenko I, Makhson A, Hartmann JT, Aparicio J, de Braud F, et al. Fluorouracil, Leucovorin, and Oxaliplatin With and Without Cetuximab in the First-Line Treatment of Metastatic Colorectal Cancer. J Clin Oncol (2009) 27:663-715. doi: 10.1200/jco.2008.20.8397

82. Aranda E, Valladares M, Martinez-Villacampa M, Benavides M, Gomez A, Massutti B, et al. Randomized Study of Weekly Irinotecan Plus High-Dose 5Fluorouracil (FUIR) Versus Biweekly Irinotecan Plus 5-Fluorouracil/ Leucovorin (FOLFIRI) as First-Line Chemotherapy for Patients With Metastatic Colorectal Cancer: A Spanish Cooperative Group for the Treatment of Digestive Tumors Study. Ann Oncol (2009) 20:251-75. doi: 10.1093/annonc/mdn557

83. Sobrero AF, Maurel J, Fehrenbacher L, Scheithauer W, Abubakr YA, Lutz MP, et al. Epic: Phase III Trial of Cetuximab Plus Irinotecan After Fluoropyrimidine and Oxaliplatin Failure in Patients With Metastatic Colorectal Cancer. J Clin Oncol (2008) 26:2311-95. doi: 10.1200/jco.2007.13.1193

84. Rothenberg ML, Cox JV, Butts C, Navarro M, Bang YJ, Goel R, et al. Capecitabine Plus Oxaliplatin (XELOX) Versus 5-Fluorouracil/Folinic Acid Plus Oxaliplatin (FOLFOX-4) as Second-Line Therapy in Metastatic Colorectal Cancer: A Randomized Phase III Noninferiority Study. Ann Oncol (2008) 19:1720-65. doi: 10.1093/annonc/mdn370
85. Hochster HS, Hart LL, Ramanathan RK, Childs BH, Hainsworth JD, Cohn AL, et al. Safety and Efficacy of Oxaliplatin and Fluoropyrimidine Regimens With or Without Bevacizumab as First-Line Treatment of Metastatic Colorectal Cancer: Results of the TREE Study. J Clin Oncol (2008) 26:3523-95. doi: 10.1200/jco.2007.15.4138

86. Heinemann V, Fischer von Weikersthal L, Vehling-Kaiser U, Stauch M, Oruzio D, Schulze M, et al. Randomized Trial Comparing Cetuximab Plus XELIRI Versus Cetuximab Plus XELOX as First Line Treatment of Patients With Metastatic Colorectal Cancer (mCRC): A Study of the German AIO CRC Study Group. J Clin Oncol (2008) 26:1865. doi: 10.1200/ jco.2008.26.15_suppl.4033

87. Haller DG, Rothenberg ML, Wong AO, Koralewski PM, Miller WH Jr, Bodoky G, et al. Oxaliplatin Plus Irinotecan Compared With Irinotecan Alone as Second-Line Treatment After Single-Agent Fluoropyrimidine Therapy for Metastatic Colorectal Carcinoma. J Clin Oncol (2008) 26:4544-505. doi: 10.1200/jco.2008.17.1249

88. Cassidy J, Clarke S, Diaz-Rubio E, Scheithauer W, Figer A, Wong R, et al. Randomized Phase III Study of Capecitabine Plus Oxaliplatin Compared With Fluorouracil/Folinic Acid Plus Oxaliplatin as First-Line Therapy for Metastatic Colorectal Cancer. J Clin Oncol (2008) 26:2006-125. doi: 10.1200/ jco.2007.14.9898

89. Borner M, Koeberle D, Von Moos R, Saletti P, Rauch D, Hess V, et al. Adding Cetuximab to Capecitabine Plus Oxaliplatin (XELOX) in First-Line Treatment of Metastatic Colorectal Cancer: A Randomized Phase II Trial of the Swiss Group for Clinical Cancer Research SAKK. Ann Oncol (2008) 19:1288-925. doi: 10.1093/annonc/mdn058

90. Saltz LB, Lenz HJ, Kindler HL, Hochster HS, Wadler S, Hoff PM, et al. Randomized Phase II Trial of Cetuximab, Bevacizumab, and Irinotecan Compared With Cetuximab and Bevacizumab Alone in IrinotecanRefractory Colorectal Cancer: The BOND-2 Study. J Clin Oncol (2007) 25:4557-615. doi: 10.1200/jco.2007.12.0949

91. Porschen R, Arkenau HT, Kubicka S, Greil R, Seufferlein T, Freier W, et al. Phase III Study of Capecitabine Plus Oxaliplatin Compared With Fluorouracil and Leucovorin Plus Oxaliplatin in Metastatic Colorectal Cancer: A Final Report of the Aio Colorectal Study Group. J Clin Oncol (2007) 25:4217-235. doi: 10.1200/jco.2006.09.2684

92. Falcone A, Ricci S, Brunetti I, Pfanner E, Allegrini G, Barbara C, et al. Phase III Trial of Infusional Fluorouracil, Leucovorin, Oxaliplatin, and Irinotecan (FOLFOXIRI) Compared With Infusional Fluorouracil, Leucovorin, and Irinotecan (FOLFIRI) as First-Line Treatment for Metastatic Colorectal Cancer: The Gruppo Oncologico Nord Ovest. J Clin Oncol (2007) 25:1670-65. doi: 10.1200/jco.2006.09.0928

93. Souglakos J, Androulakis N, Syrigos K, Polyzos A, Ziras N, Athanasiadis A, et al. FOLFOXIRI (Folinic Acid, 5-Fluorouracil, Oxaliplatin and Irinotecan) vs FOLFIRI (Folinic Acid, 5-Fluorouracil and Irinotecan) as First-Line Treatment in Metastatic Colorectal Cancer (MCC): A Multicentre Randomised Phase III Trial From the Hellenic Oncology Research Group (HORG). Br J Cancer (2006) 94:798-8055. doi: 10.1038/sj.bjc.6603011

94. Hospers GA, Schaapveld M, Nortier JW, Wils J, van Bochove A, de Jong RS, et al. Randomised Phase III Study of Biweekly 24-H Infusion of High-Dose 5FU With Folinic Acid and Oxaliplatin Versus Monthly Plus 5-FU/Folinic Acid in First-Line Treatment of Advanced Colorectal Cancer. Ann Oncol (2006) 17:443-95. doi: 10.1093/annonc/mdj104

95. Goldberg RM, Sargent DJ, Morton RF, Fuchs CS, Ramanathan RK, Williamson SK, et al. Randomized Controlled Trial of Reduced-Dose Bolus Fluorouracil Plus Leucovorin and Irinotecan or Infused Fluorouracil Plus Leucovorin and Oxaliplatin in Patients With Previously Untreated Metastatic Colorectal Cancer: A North American Intergroup Trial. J Clin Oncol (2006) 24:3347-535. doi: 10.1200/jco.2006.06.1317

96. Polikoff J, Mitchell EP, Badarinath S, Graham CD, Jennis A, Chen TT, et al. Erbitux (Cetuximab) Plus FOLFOX for Colorectal Cancer (EXPLORE): Preliminary Efficacy Analysis of a Randomized Phase III Trial. J Clin Oncol (2005) 23:2645. doi: 10.1200/jco.2005.23.16_suppl.3574

97. Kalofonos HP, Aravantinos G, Kosmidis P, Papakostas P, Economopoulos $\mathrm{T}$, Dimopoulos $\mathrm{M}$, et al. Irinotecan or Oxaliplatin Combined With Leucovorin and 5-Fluorouracil as First-Line Treatment in Advanced Colorectal Cancer: A Multicenter, Randomized, Phase II Study. Ann Oncol (2005) 16:869-775. doi: 10.1093/annonc/mdi193 
98. Comella P, Massidda B, Filippelli G, Palmeri S, Natale D, Farris A, et al. Oxaliplatin Plus High-Dose Folinic Acid and 5-Fluorouracil I.V. Bolus (OXAFAFU) Versus Irinotecan Plus High-Dose Folinic Acid and 5Fluorouracil I.V. Bolus (IRIFAFU) in Patients With Metastatic Colorectal Carcinoma: A Southern Italy Cooperative Oncology Group Phase III Trial. Ann Oncol (2005) 16:878-86. doi: 10.1093/annonc/mdi185

99. Colucci G, Gebbia V, Paoletti G, Giuliani F, Caruso M, Gebbia N, et al. Phase III Randomized Trial of FOLFIRI Versus FOLFOX4 in the Treatment of Advanced Colorectal Cancer: A Multicenter Study of the Gruppo Oncologico Dell'italia Meridionale. J Clin Oncol (2005) 23:4866755. doi: $10.1200 /$ jco.2005.07.113

100. Tournigand C, Andre T, Achille E, Lledo G, Flesh M, Mery-Mignard D, et al. FOLFIRI Followed by FOLFOX6 or the Reverse Sequence in Advanced Colorectal Cancer: A Randomized GERCOR Study. J Clin Oncol (2004) 22:229-375. doi: 10.1200/jco.2004.05.113

101. Hurwitz H, Fehrenbacher L, Novotny W, Cartwright T, Hainsworth J, Heim W, et al. Bevacizumab Plus Irinotecan, Fluorouracil, and Leucovorin for Metastatic Colorectal Cancer. N Engl J Med (2004) 350:2335-425. doi: 10.1056/NEJMoa032691

102. Goldberg RM, Sargent DJ, Morton RF, Fuchs CS, Ramanathan RK, Williamson SK, et al. A Randomized Controlled Trial of Fluorouracil Plus Leucovorin, Irinotecan, and Oxaliplatin Combinations in Patients With Previously Untreated Metastatic Colorectal Cancer. J Clin Oncol (2004) 22:23-305. doi: 10.1200/jco.2004.09.046

103. Cunningham D, Humblet Y, Siena S, Khayat D, Bleiberg H, Santoro A, et al. Cetuximab Monotherapy and Cetuximab Plus Irinotecan in IrinotecanRefractory Metastatic Colorectal Cancer. N Engl J Med (2004) 351:337455. doi: 10.1056/NEJMoa033025

104. Rothenberg ML, Oza AM, Bigelow RH, Berlin JD, Marshall JL, Ramanathan RK, et al. Superiority of Oxaliplatin and Fluorouracil-Leucovorin Compared With Either Therapy Alone in Patients With Progressive Colorectal Cancer After Irinotecan and Fluorouracil-Leucovorin: Interim Results of a Phase III Trial. J Clin Oncol (2003) 21:2059-695. doi: 10.1200/jco.2003.11.126

105. Rougier P, Lepille D, Bennouna J, Marre A, Ducreux M, Mignot L, et al. Antitumor Activity of Three Second-Line Treatment Combinations in Patients With Metastatic Colorectal Cancer After Optimal 5-FU Regimen Failure: A Randomised, Multicentre Phase II Study. Ann Oncol (2002) 13:1558-675. doi: 10.1093/annonc/mdf259

106. Maiello E, Gebbia V, Giuliani F, Paoletti G, Gebbia N, Cigolari S, et al. 5Fluorouracil and Folinic Acid With or Without CPT-11 in Advanced Colorectal Cancer Patients: A Multicenter Randomised Phase II Study of the Southern Italy Oncology Group. Ann Oncol (2000) 11:1045-515. doi: 10.1023/A:1008342928408

107. de Gramont A, Figer A, Seymour M, Homerin M, Hmissi A, Cassidy J, et al. Leucovorin and Fluorouracil With or Without Oxaliplatin as First-Line Treatment in Advanced Colorectal Cancer. J Clin Oncol (2000) 18:2938-475. doi: $10.1200 /$ jco.2000.18.16.2938

108. Nct. Irinotecan and Cetuximab With or Without Bevacizumab in Treating Patients With RAS Wild-Type Locally Advanced or Metastatic Colorectal Cancer That Cannot be Removed by Surgery (2021). Available at: https:// clinicaltrials.gov/show/nct02292758.

109. Nct. Cetuximab, Bevacizumab \& 5 fu/Leucovorin vs. Oxaliplatin, Bevacizumab \& 5FU/Leucovorin in Metastatic Colorectal Cancer (2021). Available at: https://clinicaltrials.gov/show/nct00252564. [Accessed date: June 30, 2021]

110. Nct. Study Evaluating the Safety and Efficacy of FOLFIRI Plus Cetuximab or FOLFOX Plus Cetuximab as First-Line Therapy in Subjects With KRAS WildType Metastatic Colorectal Cancer (APEC-Study) (APEC) (2021). Available at: https://clinicaltrials.gov/show/nct00778830. [Accessed date: June 30, 2021]

111. Nct. Safety and Efficacy Study of mFOLFOX6 + Panitumumab Combination Therapy and 5-FU/LV + Panitumumab Combination Therapy in Participants With Chemotherapy-Naïve Unresectable Advanced Recurrent Colorectal Carcinoma (SAPPHIRE) (2021). Available at: https://clinicaltrials.gov/show/ nct02337946. [Accessed date: June 30, 2021]

112. Nct. A Study of Avastin (Bevacizumab) in Combination With XELOX or FOLFOX-4 in Patients With Metastatic Colorectal Cancer (2021). Available at: https://clinicaltrials.gov/show/nct00349336. [Accessed date: June 30, 2021]

113. Nct. A Study of Avastin (Bevacizumab) in Combination Chemotherapy in Patients With Metastatic Cancer of the Colon or Rectum (2021). Available at: https://clinicaltrials.gov/show/nct01131078. [Accessed date: June 30, 2021]

114. Nct. Study of Bevacizumab + mFOLFOX6 Versus Bevacizumab + FOLFIRI With Biomarker Stratification in Participants With Previously Untreated Metastatic Colorectal Cancer (mCRC) (2021). Available at: https:// clinicaltrials.gov/show/nct01374425. [Accessed date: June 30, 2021]

115. Nct. Comparison of Treatment Effect of Chemotherapy With Panitumumab to Chemotherapy Alone (2021). Available at: https://clinicaltrials.gov/show/ nct00339183. [Accessed date: June 30, 2021]

116. Nct. ASPECCT: A Study of Panitumumab Efficacy and Safety Compared to Cetuximab in Patients With KRAS Wild-Type Metastatic Colorectal Cancer (2021). Available at: https://clinicaltrials.gov/show/nct01001377. [Accessed date: June 30, 2021]

117. Nct. Sequential and Concurrent FOLFOXIRI/Bevacizumab Regimens Versus Folfox/Bevacizumab in First-Line Metastatic Colorectal Cancer (2021). Available at: https://clinicaltrials.gov/show/nct01765582. [Accessed date: June 30, 2021]

118. Nct. A Trial to Compare Oxaliplatin, Folinic Acid (FA) and 5-Fluorouracil (5fu) Combination Chemotherapy (FOLFOX-4) With or Without Cetuximab in the 1st Line Treatment of Metastatic Colorectal Cancer $(m C R C)$ in Chinese Rat Sarcoma Viral Oncogene Homolog (RAS) Wild-Type Patients (2021). Available at: https://clinicaltrials.gov/show/nct01228734. [Accessed date: June 30, 2021]

119. Pfizer. Highlights of Prescribing Information: Camptosar (2014). Available at: https://www.accessdata.fda.gov/drugsatfda_docs/label/2014/020571s048lbl. pdf. [Accessed date: July 27, 2021]

120. Sanofi-Aventis. Highlights of Prescribing Information: Eloxatin (2011). Available at: https://www.accessdata.fda.gov/drugsatfda_docs/label/2009/ 021492s011,021759s009lbl.pdf. [Accessed date: July 27, 2021]

121. Genetech I. Highlights of Prescribing Information: Avastin (2017). Available at: https://www.accessdata.fda.gov/drugsatfda_docs/label/2014/ 125085s301lbl.pdf. [Accessed date: July 27, 2021]

122. Amgen I. Highlights of Prescribing Information: Vectibix (2009). Available at: https://www.accessdata.fda.gov/drugsatfda_docs/label/2009/125147s080lbl. pdf. [Accessed date: July 27, 2021]

123. Eli L. Highlights of Prescribing Information: Erbitux (2012). Available at: https://www.accessdata.fda.gov/drugsatfda_docs/label/2012/125084s0228lbl. pdf. [Accessed date: July 27, 2021]

124. Xie YH, Chen YX, Fang JY. Comprehensive Review of Targeted Therapy for Colorectal Cancer. Signal Transduct Target Ther (2020) 5:225. doi: 10.1038/ s41392-020-0116-Z

125. Grothey A, Fakih M, Tabernero J. Management of BRAF-Mutant Metastatic Colorectal Cancer: A Review of Treatment Options and Evidence-Based Guidelines. Ann Oncol (2021) 32:959-675. doi: 10.1016/ j.annonc.2021.03.206

126. Vo TT, Porcher R, Chaimani A, Vansteelandt S. A Novel Approach for Identifying and Addressing Case-Mix Heterogeneity in Individual Participant Data Meta-Analysis. Res Synth Methods (2019) 10:582-965. doi: $10.1002 /$ jrsm. 1382

127. Vo TT, Porcher R, Vansteelandt S. Assessing the Impact of Case-Mix Heterogeneity in Individual Participant Data Meta-Analysis: Novel Use of I2 Statistic and Prediction Interval. Res Methods Med Health Sci (2021) 2:12305. doi: $10.1177 / 2632084320957207$

128. Jansen JP. Network Meta-Analysis of Individual and Aggregate Level Data. Res Synth Methods (2012) 3:177-905. doi: 10.1002/jrsm.1048

129. Saramago P, Sutton AJ, Cooper NJ, Manca A. Mixed Treatment Comparisons Using Aggregate and Individual Participant Level Data. Stat Med (2012) 31:3516-365. doi: 10.1002/sim.5442

Conflict of Interest: The authors declare that the research was conducted in the absence of any commercial or financial relationships that could be construed as a potential conflict of interest.

Publisher's Note: All claims expressed in this article are solely those of the authors and do not necessarily represent those of their affiliated organizations, or those of the publisher, the editors and the reviewers. Any product that may be evaluated in this article, or claim that may be made by its manufacturer, is not guaranteed or endorsed by the publisher. 
Copyright (c) 2022 Hoang, Sohn, Kim, Cha and Kim. This is an open-access article distributed under the terms of the Creative Commons Attribution License (CC BY).

The use, distribution or reproduction in other forums is permitted, provided the original author(s) and the copyright owner(s) are credited and that the original publication in this journal is cited, in accordance with accepted academic practice. No use, distribution or reproduction is permitted which does not comply with these terms. 